Article

\title{
Complements to Mügge and Friedel's Theory of Twinning
}

\section{Cyril Cayron}

LMTM, EPFL (Ecole Polytechnique Fédérale de Lausanne), PX Group Chair, 2000 Neuchâtel, Switzerland; cyril.cayron@epfl.ch

Received: 14 January 2020; Accepted: 4 February 2020; Published: 7 February 2020

\begin{abstract}
The crystallography of twinning is based on the concepts of simple shear and obliquity introduced by Mügge, Mallard and Friedel at the turn of the last century, with tensor mathematics later developed by Bilby, Bevis and Crocker in the 1960s. We propose a synthesis of these works by writing the three transformations (distortion, orientation and correspondence) as matrices in dyadic product forms. We show that a "normal" Friedelian mode is implicitly assumed. We introduce another mode called "tilted" that explains, with the simple twin index $q=1$, some twins that were previously oddly reported with $q=2$. We also interpret the type II twins, which are usually presented as the conjugate twins of type I twins, as simple shears a rational reciprocal plane, exactly as the type I twins are simple shears a rational direct plane. Finally, we explain why the term "twin" for variants inherited from a phase transformation is not appropriate, and we call for a generalization of the crystallography of twinning by considering epitaxial distortions and iso-orientation shears.
\end{abstract}

Keywords: twins; shear; lattice; metrics; crystallography

\section{Introduction}

The research for a general theory of twinning dates from the early days of crystallography, as detailed in an excellent book [1]. The existence of the lattice was discovered by Haüy, but the notion of atoms was not fully accepted, and the "motif" associated with the lattice could just be imagined as a continuum medium filling the unit cell. Haüy and Bravais noticed that the interfaces of growth twins in gemstones are often flat and follow low-index planes [2]. Similar observations were made on deformation twins in calcite and other minerals. Deformation twinning was thus imagined to result from a simple shear of lattice [3]. The shear should be special in order to "restore" the lattice of the initial phase. In the 1880s, Mügge [4,5] found the general equations to describe the orientation relationship between a crystal and its twin formed by deformation. He introduced the important concepts of type I and type II twins thanks to the four geometrical elements of twinning $\left(\mathbf{K}_{1}, \eta_{1}\right.$, $\mathbf{K}_{2}, \boldsymbol{\eta}_{2}$ ), with the reticular plane $\mathbf{K}_{1}$ that contains a direction $\boldsymbol{\eta}_{1}$ (not necessarily reticular), and their conjugates, the plane $\mathbf{K}_{2}$ (not necessarily reticular) that contains the reticular direction $\eta_{2}$ (see also [6] for the historic details). In this paper, we will use equivalently the terms "reticular" and "rational" to mean that a plane or a direction belongs to the lattice, i.e., their coordinates are integers for simple lattices, and half-integers for body-centered cubic (bcc) or face-centered cubic (fcc) lattices. Type I twins are defined by their shear plane $\boldsymbol{p}=\mathbf{K}_{1}$ along the shear direction $\mathbf{d} / / \boldsymbol{\eta}_{1}$ with an amplitude $s$, as shown in Figure 1a. This shear leaves both the direction $\eta_{2}$ and the plane $\mathbf{K}_{2}$ undistorted but rotated by an angle $2 \theta$. The angle $\theta$ is linked to the shear amplitude by $s=2 \tan (\theta)$. Type II twins are defined from type I twins by exchanging $\left(\mathbf{K}_{1}, \boldsymbol{\eta}_{1}\right)$ with $\left(\mathbf{K}_{2}, \boldsymbol{\eta}_{2}\right)$, i.e., such that the shear plane is $\boldsymbol{p}=\mathbf{K}_{2}$ along the shear direction $\mathbf{d} / / \boldsymbol{\eta}_{2}$. In other words, type I twins are defined by a rational shear plane, and type II twins by a rational shear direction [7-10]. When the four elements are rational the twin is said to be "compound". All the twins in cubic crystals are compound twins. For a type I twin, $\mathbf{K}_{1}$ is a mirror 
plane for the twin edifice; it is also the interface between the two individual crystals. For type II twins, the direction $\eta_{2}$ is a $180^{\circ}$ rotation for the edifice. Many growth twins of type II do not exhibit a clear straight interface, or if it exists, it is the plane $\mathbf{K}_{2}$ or a rational plane closest to $\mathbf{K}_{2}$ containing $\eta_{2}$. It is assumed that the plane $\mathbf{K}_{1}$ for type I twins and the direction $\eta_{2}$ for type II twins cannot be symmetry elements of the individual crystals. After Mügge, the mathematics inherited from these concepts were developed by Kihô [11], Jaswon and Dove [12], and later by Bilby, Bevis and Crocker [13-15].

In parallel to Mügge's work on deformation twins, Mallard [16] introduced important concepts for the crystallography of growth twins in the period 1876-1886: (a) "twinning by merohedry" where the crystal and its twin share the same lattice but the orientation of the motif is different; and (b) "twinning by pseudo merohedry" in which a reticular plane is "nearly" a mirror plane of the crystal or a reticular direction is nearly a $180^{\circ}$ rotation (see also $[17,18]$ ). A plane can become a mirror symmetry for the twin edifice when its normal is close to a reticular direction, and a direction can become a $180^{\circ}$ rotation when its normal plane is close to a reticular plane. In such cases, the lattice of the crystal and that of its twin are "close", which means that a slight distortion is sufficient to transform one into the other. An example is shown in Figure $1 \mathbf{b}$. The direction $\mathbf{n}$ normal to the plane $\mathbf{K}_{1}=(0,1)$ is close to the reticular direction $\eta_{2}=[0,1]$; twinning can occur because the obliquity $\theta$, i.e., the misorientation angle between the two directions, is small. The parent and twin lattices are in mirror orientation through their common $\mathbf{K}_{1}$ plane. We took the liberty to use here Mügge's notations of deformation twinning $\mathrm{K}_{1}$ and $\eta_{2}$, whereas Friedel never used them to describe the growth twins. Note that the unit cell rhombi of the cell can be imagined as derived from an hypothetical square, and that the $(0,1)$ plane would be a symmetry element of this square. Mallard believed that the existence of the pseudo-symmetry necessarily involves an imaginary phase of higher symmetry that would act as a parent phase for both the crystal and its twin, but Friedel disagreed on this point and clearly stated that twinning is not a matter of symmetry but of metrics [19].

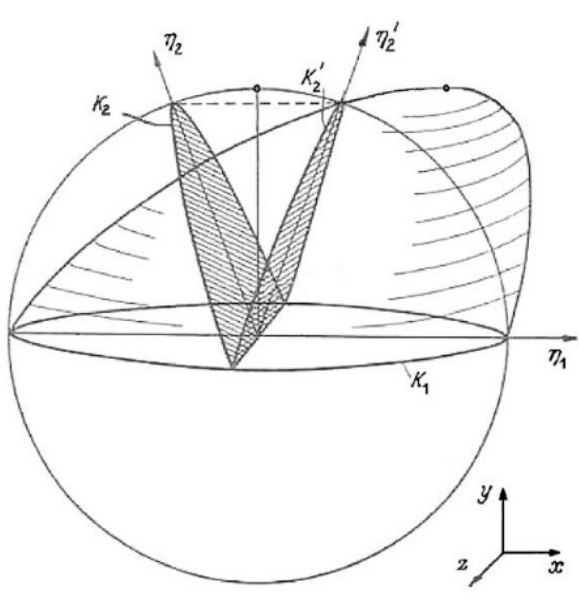

(a)

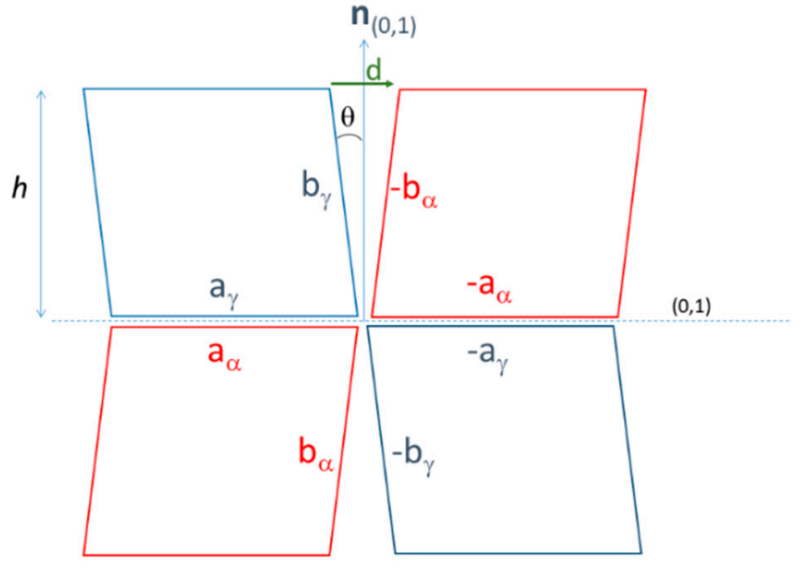

(b)

Figure 1. Similarities between the models of deformation and growth twinning shown in the case of type I twins. (a) Deformation twinning by simple shear introduced by Mügge in 1889 and represented by Hall in 1954. Reprinted from [9] with permission from Elsevier. The angle between the plane $\mathbf{K}_{2}$ and its image $\mathbf{K}_{2}{ }^{\prime}$ by the shear along $\mathbf{d} / / \boldsymbol{\eta}_{1}$ is $2 \theta$ (d and $\theta$ are not marked in the figure). (b) $2 \mathrm{D}$ representation of twinning by pseudo-merohedry as initially introduced by Mallard in 1893 and Friedel in 1904 for growth twins. The parent lattice is in blue and its twin in red. The obliquity $\theta$ is the angle between the reticular direction $\eta_{2}=\mathbf{b}=[0,1]$ and the normal to the plane $\mathbf{K}_{1}=(0,1)$. The two lattices are in mirror symmetry and differ by a slight misfit represented by the vector $\mathbf{d} / /[1,0]$.

Friedel introduced in 1904 the concept of "twinning by reticular pseudo merohedry" by generalizing Mallard's concept of "pseudo-merohedry" to the "multiple" lattices. The ratio of 
the volume of the supercell that forms the multiple lattice divided by that of the unit cell is an integer called "twin index" $[19,20]$ and noted $q$. The Coincidence Site Lattice (CSL) and the associated number $\Sigma$ introduced by Bollmann in 1970 [21] are nothing else than the multiple lattice and twin index introduced by Friedel decades earlier (see [22] for historical details). Mallard and Friedel's theory has strongly influenced mineralogy because it explains many growth twins observed in numerous gemstones and rocks.

Friedel did not clearly recognize that the concept of "obliquity" he introduced for the growth twins is in direct correspondence with that of shear introduced by Mügge two decades earlier, even if he perfectly knew and largely cited Mügge's works on deformation twinning in 1926 [20]. However, as already mentioned, both are directly linked by $s=d / h=2 \tan (\theta)$. This relation is important because it means that a unique crystallographic model could explain both the growth twins and the deformation twins. For deformation twins, experiments show that only the twins with the lowest shear values are observed. This result is so well known that it is perceived as "obvious"; but it is not. Actually, the twins with high shear values would relax more efficiently the applied stress than low shear twins, and they should thus be favored by thermodynamics because the Gibbs free energy $G$ is reduced by the external work $\mathrm{W}=\boldsymbol{\sigma}_{i j} \boldsymbol{\varepsilon}_{i j}$. However, a large shear twin cannot form instantaneously in the whole volume of the sample; accommodation in the external surrounding parent host is required, which introduces a positive term to G. Only the contribution of the accommodation term explains why the observed twins are generally low shear twins. The physical origin of the growth twins is different. They appear at the first stage of the formation of a crystal as accidental defects that are made possible only because of the low energy of the interface between the crystal and its twin. In general, the crystal grows perpendicularly to low energy faces that are often planes with low indices. If the growth plane is not a symmetry plane of the crystal, the stacking is faulted and this generates a newly oriented crystal, i.e., a twin. The crystal and the twin then grow at the same speed to form the twinned edifice. If the twin could be formed instantaneously on perfectly flat layer, the value of the obliquity would have no effect on the propensity of the twin to form. As shown by Friedel, however, only twins with low obliquity are observed. This is because the interface is never perfectly flat and the twin co-exists with its parent crystal in the volume. Therefore, as for deformation twins, the accommodation of the distortion between the two lattices is important and should be considered in three dimensions. This qualitatively explains why the same crystallographic approach can work for both deformation and growth twins despite the difference of mechanism. The misfit to accommodate the formation of growth twins is quite similar to the shear distortion of deformation twins. In his excellent 1954 review, Cahn [7] noticed that the shear associated with some deformation twins in metals is very large (up to $0.7)$, implying an obliquity $\left(19^{\circ}\right)$ far larger than the maximum admitted obliquity for growth twins $\left(\sim 6^{\circ}\right)$. This fact has discouraged crystallographers to develop a unified theory. Mineralogists continue their research on growth twins with Friedel's theory, whereas metallurgists and geologists interested in the mechanical properties use Mügge, Bilby, Bevis and Crocker equations. However, to our opinion, the mathematics is the same for both types of twins.

The manuscript aims at presenting a synthetic and didactic view on the crystallography of twins. It will explain in details how the three transformation matrices (shear, orientation and correspondence) disseminated until now under various forms in a vast literature can be determined and expressed uniformly as dyadic products. This systematic approach will be helpful to realize that the earlier works rely on Friedel's assumption of "low obliquity" that implicitly links the orientation and the shear matrices. A new twin mode that breaks this correlation, called "tilted" mode, will be introduced to complete the "normal" Friedelian mode. It will allow us to explain with a simple twin index $q=1$ some deformation twins in magnesium and bcc metals oddly reported in earlier literature with $q=2$. Another contribution of the paper concerns the type II twins. We will show that they can be introduced independently of the type I twins as simple shears acting on a rational plane of the reciprocal space. Eventually, we will discuss why we think that the terminology "transformation twins" for phase 
transformation variants is inappropriate. We will also explain why the crystallography of twins should continue to evolve by exploring hypotheses that were forbidden, more by dogmatism than by physics.

\section{The Type I Twins and the Twinning Transformation Matrices}

\subsection{Crystallographic Notations}

Jaswon, Dove, Bilby, Bevis and Crocker and other authors used the tensor notation with the full expression of the indices that makes the reading of their papers uneasy. Matrix notation in which the indices are not written allow lighter equations, as already commented by Christian and Mahajan [10]. Here, we will use the same matrix notations and conventions as in Reference [23]. We briefly recall them. Vectors are written in bold lower cases, and matrices in bold capital letters. A vector $\mathbf{d}$ of the direct space is in column, and a vector $\mathbf{p}^{*}$ of the reciprocal space is in line. The same reciprocal vector is simply written $\mathbf{p}$ when it is in column, i.e., $\mathbf{p}^{*}=\mathbf{p}^{t}$ with the symbol $t$ in the superscript meaning "transpose" (not "twin"). A term-by-term scalar product is calculated by taking a vector of the reciprocal space and a vector of the direct space, for example $\mathbf{p}^{*} \cdot \mathbf{d}=\mathbf{p}^{\mathbf{t}} \cdot \mathbf{d}=\mathbf{p}_{i} \mathbf{d}_{i}$ with Einstein's convention, i.e., summing the coordinates of indices $i \in\{1,2,3\}$. The dyadic product of the vectors $\mathbf{d} \otimes \mathbf{p}=\left(\mathbf{d} \cdot \mathbf{p}^{\mathrm{t}}\right)$ is the matrix $\mathbf{d}_{i} \mathbf{p}_{j}$. By default, we will always write the equations with column vectors. The dyadic product notation has all the properties of the matrix product; it is for example associative, which means that for any vector $\mathbf{u}$, we get $\left(\mathbf{d} \cdot \mathbf{p}^{\mathrm{t}}\right) \cdot \mathbf{u}=\mathbf{d} \cdot\left(\mathbf{p}^{\mathrm{t}} \cdot \mathbf{u}\right)$, i.e., it is the vector $\mathbf{d}$ multiplied by the scalar product $\mathbf{p}^{\mathbf{t}} \cdot \mathbf{u}$.

We recall that if a distortion matrix $\mathbf{F}$ acts on the vectors $\mathbf{u}$ of the direct space, $\mathbf{d}^{\prime}=\mathbf{F} \cdot \mathbf{d}$, the same distortion acts on the plane $\mathbf{p}$ by $\mathbf{p}^{\prime}=\mathbf{F}^{*} \cdot \mathbf{p}$, with $\mathbf{F}^{*}=\mathbf{F}^{-\mathbf{t}}$ (inverse of the transpose of $\mathbf{F}$ ). It can be checked that any direction $\mathbf{d}$ lying on the plane $\mathbf{p}$ remains on the plane after distortion as $\mathbf{p}^{\prime \mathrm{t}} \cdot \mathbf{d}^{\prime}=\left(\mathbf{F}^{-\mathrm{t}} \mathbf{p}\right)^{\mathrm{t}} \cdot \mathbf{F d}=\mathbf{p}^{\mathrm{t}} \cdot \mathbf{d}=0$. For any crystal one can be associate a crystallographic basis $\mathfrak{B}_{c}=(\mathbf{a}, \mathbf{b}, \mathbf{c})$ formed by the usual crystallographic vectors. At the basis $\mathfrak{B}_{c}$ can be associated a $3 \times 3$ unit cell matrix $\mathbf{B}_{c}=[\mathbf{a}, \mathbf{b}, \mathbf{c}]$ by writing the coordinates of $\mathbf{a}, \mathbf{b}, \mathbf{c}$ in columns in a unit orthonormal reference frame. The metrics of the crystal is then defined by the metric tensor $\mathcal{M}=\mathbf{B}_{c}^{\mathrm{t}} \mathbf{B}_{c}=\left[\begin{array}{ccc}\mathbf{a}^{2} & \mathbf{b}^{\mathrm{t}} \cdot \mathbf{a} & \mathbf{c}^{\mathrm{t}} \cdot \mathbf{a} \\ \mathbf{a}^{\mathrm{t}} \cdot \mathbf{b} & \mathbf{b}^{2} & \mathbf{c}^{\mathrm{t}} \cdot \mathbf{b} \\ \mathbf{a}^{\mathrm{t}} \cdot \mathbf{c} & \mathbf{b}^{\mathrm{t}} \cdot \mathbf{c} & \mathbf{c}^{2}\end{array}\right]$. The metric tensor is nothing else than the coordinate transformation matrix between the reciprocal space and the direct space $\mathcal{M}=\left[\boldsymbol{B}_{c}^{*} \rightarrow \mathfrak{B}_{c}\right]$. It has the properties to be symmetric $\mathcal{M}=\mathcal{M}^{\mathrm{t}}$, and $\mathcal{M}^{*}=\left[\mathfrak{B}_{c} \rightarrow \mathfrak{B}_{c}^{*}\right]=\mathcal{M}^{-1}$. The scalar product between the vectors $\mathbf{u}$ and $\mathbf{v}$ of the direct space is determined by expressing one vector in the reciprocal space thanks to the metric tensor, $(\mathbf{u} \cdot \mathbf{v})=\mathbf{u}^{\mathrm{t}} \mathcal{M} \mathbf{v}$. The norm $\|\mathbf{d}\|$ of a vector $\mathbf{d}$ of the direct space, and the norm $\|\mathbf{p}\|^{*}$ of a vector $\mathbf{p}$ of the reciprocal space are respectively given by:

$$
\|\mathbf{d}\|=\sqrt{\mathbf{d}^{\mathrm{t}} \mathcal{M} \mathbf{d}} \text { and }\|\mathbf{p}\|^{*}=\sqrt{\mathbf{p}^{\mathrm{t}} \mathcal{M}^{*} \mathbf{p}}
$$

The notation $\tilde{\mathbf{d}}$ applied to a direct vector means that $\mathbf{d}$ is normalized by $\|\mathbf{d}\|$, and the notation $\tilde{\mathbf{p}}$ applied to a reciprocal vector $\mathbf{p}$ means that $\mathbf{p}$ is normalized by $\|\mathbf{p}\|^{*}$.

$$
\tilde{\mathbf{d}}=\frac{\mathbf{d}}{\|\mathbf{d}\|^{*}} \text { and } \tilde{\mathbf{p}}=\frac{\mathbf{p}}{\|\mathbf{p}\|^{*}}
$$

The inter-reticular distance $d_{h k l}$ between the planes $\mathbf{p}$ of Miller indices $\boldsymbol{p}=(h, k, l)$ is:

$$
d_{h k l}=\frac{1}{\|\mathbf{p}\|^{*}}
$$

The unit normal direction in the direct space of a plane $\mathbf{p}$ is $\mathbf{n}$ given by:

$$
\mathbf{n}=\mathcal{M}^{*} \tilde{\mathbf{p}}
$$


It can be verified that $\mathbf{n}^{\mathrm{t}} \mathbf{M n}=\mathbf{n}^{\mathrm{t}} \tilde{\mathbf{p}}=1$.

The present paper is written by trying to find a good compromise between a rigorous but heavy notation and a weak but easy-to-read one. The use of the conventional crystallographic notations is respected, even if sometimes the reader must remain very careful to avoid confusion. For example, we use the conventional indices $u, v, w$ in italic for a direction $\mathbf{d}=[u, v, w]$, but we also use the same letters in bold to designate three vectors $\mathbf{u}, \mathbf{v}, \mathbf{w}$. Similarly, we use the conventional indices $h, k, l$ for a plane $\mathbf{p}=(h, k, l)$ without any relation with the reciprocal vectors $\mathbf{h}, \mathbf{k}, \mathbf{l}$. The value $h$ also designates in the text the distance between the $q$-layers of the $p$-plane.

Three matrices play key roles in the crystallographic description of structural phase transformation in general, and for deformation twinning in particular. They are the lattice distortion matrix $\mathbf{F}$, the orientation relationship matrix $\mathbf{T}$, and the correspondence matrix $\mathbf{C}$, as detailed in Reference $[23,24]$. The methods to calculate them have been progressively developed at the end of 19th and along the 20th centuries by Mügge, Kihô, Jaswon, Dove, Bilby, Bevis and Crocker. They are all based on the Friedelian notion of "normal" obliquity (as it will be clarified in Section 4).

\subsection{Schematic Representation of Twinning}

The main equations of $\mathbf{F}, \mathbf{T}$ and $\mathbf{C}$ matrices of type I twins are already reported in literature, but spread in different papers [4-15], and sometimes without clearly explaining the nature of the proposed matrix. We will give to them in a unique formalism by considering Figure 2.

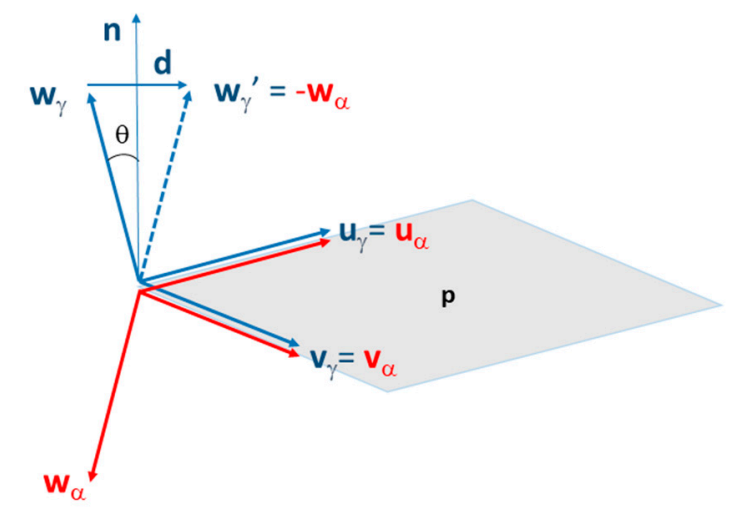

Figure 2. Schematic representation of a type I deformation twinning (normal mode). The parent crystal $\Upsilon$ is in blue and its twin $\alpha$ in red. The supercell $(\mathbf{u}, \mathbf{v}, \mathbf{w})$ of the parent crystal $\Upsilon$ is distorted by a simple shear along the direction $\mathbf{d}$ that lies in the plane $\mathbf{p}$ such that its image $\left(\mathbf{u}^{\prime}, \mathbf{v}^{\prime}, \mathbf{w}^{\prime}\right)$ becomes an equivalent supercell of the twin $\alpha$. The angle $\theta=(\mathbf{w}, \mathbf{n})=\left(\mathbf{w}^{\prime}, \mathbf{n}\right)$ is the "normal" obliquity, where $\mathbf{n}$ is the normal to the plane $\mathbf{p}$.

The parent crystal is noted $\Upsilon$, and its twin $\alpha$. Actually, they are the same phase, and the Greek letters are used here only to differentiate the orientations of the crystals. We call $\mathbf{u}_{\gamma}, \mathbf{v}_{\gamma}, \mathbf{w}_{\gamma}$ the three reticular vectors of the parent crystal forming the supercell that is sheared. Their coordinates are rational. The lower index $\curlyvee$ refers to the crystal $\Upsilon$. Please note that in our paper [23] $\curlyvee$ was written as an upper index, but here we prefer to put it to the lower position to leave space for the sign " $\mathrm{t}$ " (transpose) in the dyadic products. When the coordinates of a vector $\mathbf{u}$ are expressed in the crystallographic basis of the crystal $\gamma$, we use the notation $\mathbf{u}_{/ \gamma}$. For example $\mathbf{u}_{\alpha / \gamma}$ means a reticular vector of the crystal $\alpha$ whose coordinates are written in $\mathfrak{B}_{c}^{\gamma}$, where $\mathfrak{B}_{c}^{\gamma}$ is the crystallographic basis of the crystal $\Upsilon$. When the coordinates of reticular vectors of phase $\Upsilon$ are written in their own crystallographic basis $\mathfrak{B}_{c}^{\gamma}$, we simply write $\mathbf{u}_{\gamma}$ in place of $\mathbf{u}_{\gamma / \gamma}$; in other words we assimilate the geometrical vector $\mathbf{u}_{\gamma}$ to its algebraic coordinates $\mathbf{u}_{\gamma / \gamma}$.

We note $\mathbf{u}^{\prime}=\mathbf{u}, \mathbf{v}^{\prime}=\mathbf{v}$, and $\mathbf{w}^{\prime}$ the images of the vectors $\mathbf{u}, \mathbf{v}$, and $\mathbf{w}$ by the simple shear $\mathbf{F}$ on the plane $\mathbf{p}=(\mathbf{u}, \mathbf{v})$ along the direction $\mathbf{d}$. For type I twins, $\mathbf{p}=\mathbf{K}_{1}$ and $\mathbf{d} / / \boldsymbol{\eta}_{1}$. The direction $\mathbf{w}$ is the 
direction usually called $\eta_{2}$. It is just rotated; its length is unchanged $\|\mathbf{w}\|=\left\|\mathbf{w}^{\prime}\right\|$. It should be noted that $\mathbf{w}$ points to a node located in the $q$ th layer of plane $\mathbf{p}$, as illustrated in Figure 3 . Thus, the direction $\mathbf{w}$ and its image $\mathbf{w}^{\prime}$ are such that $\mathbf{p}^{\mathrm{t}} \cdot \mathbf{w}=\mathbf{p}^{\mathrm{t}} \cdot \mathbf{w}^{\prime}=q$, where $q$ is the twin index (integer). As $\mathbf{u}$ and $\mathbf{v}$ are reticular vectors, $q$ is also the volume of the supercell divided by the volume of the primitive cell. The case $q=1$ defines a primitive lattice, and thus applies to "twinning by pseudo merohedry", and the case $q>1$ defines a multiple lattice (a supercell) and thus applies to "twinning by reticular pseudo merohedry" in Friedel's terminology. The input for type I twins are the reticular shear plane $\mathbf{p}=\mathbf{K}_{1}$ and a reticular direction $\mathbf{w}$. Actually, the only input is $\mathbf{p}$ since $\mathbf{w}$ can be deduced from $\mathbf{p}$ by Bezout's algorithm [25].

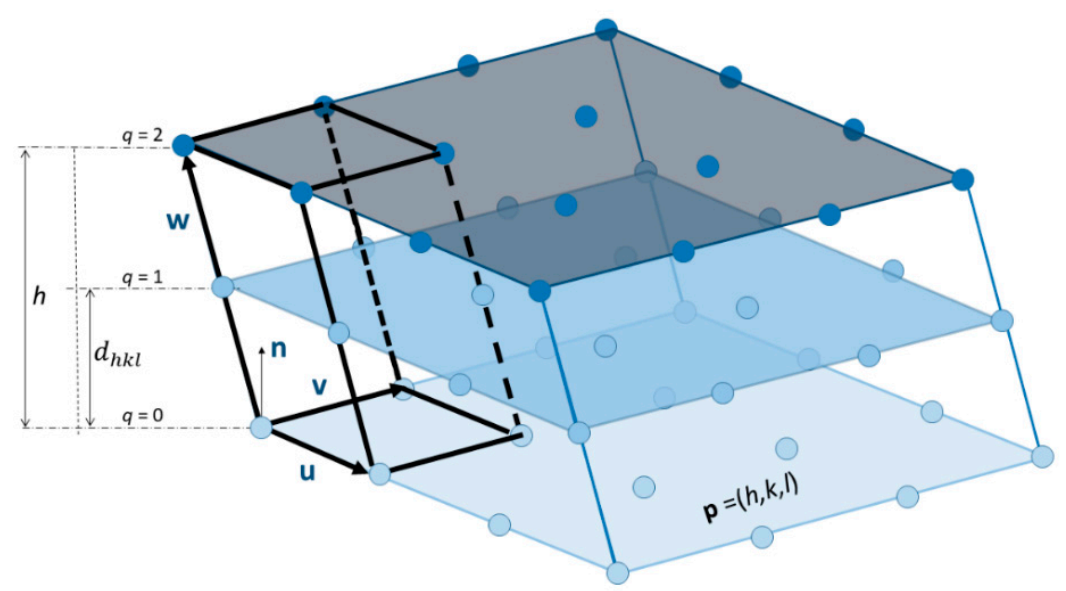

Figure 3. Schematic representation of the layers formed by the plane $\mathbf{p}=(h, k, l)$. The inter-reticular distance is noted $d_{h k l}$. The vector normal to the plane is $\mathbf{n}$ in the direct space. The nodes of the lattices are marked by small circles. The supercell of the parent crystal that is used to model the type I twins is $(\mathbf{u}, \mathbf{v}, \mathbf{w})$ marked in bold black. Here, the vector $\mathbf{w}$ points to a node of the second layer $q=2$. The distance $h$ between the $\mathbf{p}$-layers is $h=q \cdot d_{h k l}$.

\subsection{The Distortion Matrix F}

From a mechanical point of view, a simple shear can be described by two orthogonal vectors, the shear direction $\mathbf{d}$ that is a vector of the direct space, and the shear plane $\mathbf{p}$ that is a vector of the reciprocal space. They are orthogonal, which means that $\mathbf{p}^{\mathbf{t}} \cdot \mathbf{d}=0$. The matrix of simple shear is calculated from the dyadic product between the normalized vectors $\mathbf{d}$ and $\mathbf{p}$ expressed in the crystallographic basis (not specified here to make the formula more compact), that is:

$$
\mathbf{F}=\mathbf{I}+s\left(\tilde{\mathbf{d}} \cdot \tilde{\mathbf{p}}^{t}\right)
$$

with I the identity matrix, and $s$ the shear amplitude. The notation $\mathbf{F}^{\gamma}$ will be also used to specify that $F$ acts on the reticular directions of the crystal $r$.

It can be checked that for any vector $\mathbf{r}$ that belongs to $\mathbf{p}$, i.e., $\mathbf{p}^{\mathbf{t}} \cdot \mathbf{r}=0$, then $\mathbf{F} \mathbf{r}=\mathbf{r}$. In addition, $\mathbf{n}$ the unit normal to the plane $\mathbf{p}$ given by Equation (4) is transformed into the vector $\mathbf{n}^{\prime}=\mathbf{F} \mathbf{n}=\mathbf{n}+s \tilde{\mathbf{d}}$, and any vector $\mathbf{u}=\mathbf{n}+\mathbf{r}$ is transformed such that $\mathbf{u}^{\prime}-\mathbf{u}=\mathbf{n}^{\prime}-\mathbf{n}=\tilde{\mathbf{d}}$, which signifies that $s$ in Equation (5) is the measure of the displacement along the direction $\mathbf{d}$ of any point located in a plane parallel to $\mathbf{p}$ at a unit distance from $\mathbf{p}$. In order to determine $\mathbf{F}$ from Equation (5) one needs to determine the direction $\mathbf{d}$ and the shear value $s$ as function of the reticular plane $\mathbf{p}$ and reticular direction $\mathbf{w}$ used to define the supercell. The directions $\mathbf{u}, \mathbf{v}$ are left invariant by the shear, $\mathbf{u}^{\prime}=\mathbf{u}$, and $\mathbf{v}^{\prime}=\mathbf{v}$. The direction $\mathbf{w}^{\prime}=\mathbf{F} \mathbf{w}$ is such that $\mathbf{w}^{\prime}-\mathbf{w}=\mathbf{d}$ is the shear direction (not rational, at least in the usual theory), with:

$$
\mathbf{d}=-2 \mathbf{w}+2 h \mathbf{n}
$$


where $h$ is the distance between the p-layers that are sheared, $h=q \cdot d_{h k l}=\frac{q}{\|\mathbf{p}\|^{*}}$ and $h \mathbf{n}=q \frac{\mathcal{M}^{*} \mathbf{p}}{\mathbf{p}^{\mathbf{t}} \mathcal{M}^{*} \mathbf{p}}$.

Pythagorean theorem also shows that $\|\mathbf{d}\|^{2}=4\left(\|\mathbf{w}\|^{2}-h^{2}\right)$. Consequently:

$$
s^{2}=\frac{\|\mathbf{d}\|^{2}}{h^{2}}=4\left(\frac{\|\mathbf{w}\|^{2}}{h^{2}}-1\right)
$$

Equation (7) is a classical formula of literature (see for example [10]). Combining Equation (5) with (6) and (7) allows determining the shear distortion matrix only from $\mathbf{p}\left(\mathrm{K}_{1}\right)$ and $\mathbf{w}\left(\eta_{2}\right)$. We can also note that $\operatorname{Tr}(\mathbf{F})=\operatorname{Tr}(\mathbf{I})+\sin \left(\tilde{\mathbf{d}} \cdot \tilde{\mathbf{p}}^{\mathrm{t}}\right)$ with $\operatorname{Tr}\left(\tilde{\mathbf{d}} \cdot \tilde{\mathbf{p}}^{\mathrm{t}}\right)=\operatorname{Tr}\left(\tilde{\mathbf{p}}^{\mathrm{t}} \cdot \tilde{\mathbf{d}}\right)=0$. Consequently, $\operatorname{Tr}(\mathbf{F})=3$. The trace of the simple shear matrix is equal to the space dimension.

\subsection{The Orientation Matrix T}

The orientation matrix that encodes the orientation relationship between the twin and the parent crystals is the matrix $\mathbf{T}^{\alpha \rightarrow \gamma}=\left[\mathfrak{B}_{c}^{\alpha} \rightarrow \mathfrak{B}_{c}^{\gamma}\right]$. The upper index $\alpha \rightarrow \gamma$ means that the matrix transforms the coordinates of vectors written in $\mathfrak{B}_{c}^{\gamma}$ into coordinates expressed in $\mathfrak{B}_{c}^{\alpha}$ with the (left-right) $=$ (head-tail) convention for composition, for example $\mathbf{T}^{1 \rightarrow 3}=\mathbf{T}^{1 \rightarrow 2} \mathbf{T}^{2 \rightarrow 3}$. With the coordinate transformation matrix, the coordinates of the vectors $\mathbf{u}_{\gamma}, \mathbf{v}_{\gamma}$, expressed in the basis $\mathfrak{B}_{c}^{\alpha}$ are $\mathbf{u}_{\gamma / \alpha}=\mathbf{T}^{\alpha \rightarrow \gamma} \mathbf{u}_{\gamma}=\mathbf{u}_{\gamma}, \mathbf{v}_{\gamma / \alpha}=\mathbf{T}^{\alpha \rightarrow \gamma} \mathbf{v}_{\gamma}=\mathbf{v}_{\gamma}$. We can also consider the normal $\mathbf{n}$ to the plane $\mathbf{p}$ and notice that $\mathbf{n}_{\gamma / \alpha}=\mathbf{T}^{\alpha \rightarrow \gamma} \mathbf{n}_{\gamma}=-\mathbf{n}_{\gamma}$. The matrix that responds to these constraints is:

$$
\mathbf{T}^{\alpha \rightarrow \gamma}=m_{(\mathbf{p})}=\mathbf{I}-2\left(\mathbf{n} \cdot \tilde{\mathbf{p}}^{\mathrm{t}}\right)
$$

We recall that $\mathbf{n}$ is deduced from $\mathbf{p}$ by Equation (4). Thus, Equation (8) can also be written $\mathbf{T}^{\alpha \rightarrow \gamma}=\mathbf{I}-\frac{2}{\mathbf{p}^{\mathrm{t}} \mathcal{M}^{*} \mathbf{p}}\left(\mathcal{M}^{*} \mathbf{p} \cdot \mathbf{p}^{\mathrm{t}}\right)$. The orientation matrix $\mathbf{T}^{\alpha \rightarrow \gamma}$ is simply a mirror symmetry through the plane p. Following Friedel, we will consider that twinning is a matter of lattices and metrics, not of symmetry. With this hypothesis, there is not distinction between a mirror symmetry through the plane $\mathbf{p}$ and a rotation of $180^{\circ}$ around $\mathbf{n}$. This rotation is deduced from $m_{(\mathbf{p})}$ by a composition with the inversion $-\mathbf{I}$. That is:

$$
r_{\left(180^{\circ}, \mathbf{n}\right)}=-\mathbf{I}+2\left(\mathbf{n} \cdot \tilde{\mathbf{p}}^{\mathrm{t}}\right)
$$

This last equation was reported by Bilby and Crocker [13] with a more complex tensor notation. We insist again on the fact that $m_{(\mathbf{p})}$ and $r_{\left(180^{\circ}, \mathbf{n}\right)}$ are completely equivalent to describe the lattice. Whatever the choice, it can be easily checked that $\left(\mathbf{T}^{\alpha \rightarrow \gamma}\right)^{2}=\mathbf{I}$, which also implies that $\mathbf{T}^{\gamma \rightarrow \alpha}=\left(\mathbf{T}^{\alpha \rightarrow \gamma}\right)^{-1}=\mathbf{T}^{\alpha \rightarrow \gamma}$. As we decide to use the convention that type I twins are mirror symmetries for the edifice, we will continue using Equation (8). With this convention, twinning switches a right-hand basis into a left-hand basis; the determinant of the orientation matrix is $\operatorname{Det}(\mathbf{T})=-1$.

\subsection{The Correspondence Matrix C}

Taking the convention that the twinned crystal $\alpha$ is in $\mathbf{p}$-mirror symmetry relation with the parent crystal $\checkmark$, the directions $\mathbf{u}_{\gamma}, \mathbf{v}_{\gamma}, \mathbf{w}_{\gamma}$ become after distortion the directions $\mathbf{u}_{\gamma}^{\prime}=\mathbf{u}_{\alpha}, \mathbf{v}_{\gamma}^{\prime}=\mathbf{v}_{\alpha}$, $\mathbf{w}_{\gamma}^{\prime}=-\mathbf{w}_{\alpha}$. The coordinates of the vectors $\mathbf{u}_{\alpha}, \mathbf{v}_{\alpha}, \mathbf{w}_{\alpha}$ in their crystallographic basis $\mathfrak{B}_{c}^{\alpha}$ are the same as those of the vectors $\mathbf{u}_{\gamma}, \mathbf{v}_{\gamma}, \mathbf{w}_{\gamma}$ in their crystallographic basis $\mathfrak{B}_{c}^{\gamma}$. The correspondence matrix gives the coordinates of the vectors $\mathbf{u}_{\gamma}^{\prime}, \mathbf{v}_{\gamma}^{\prime}, \mathbf{w}_{\gamma}^{\prime}$ in $\mathfrak{B}_{c}^{\alpha}$, the crystallographic basis of the twin. It was initially determined as a linear form by Mügge [4], latter corrected by Pabst [26], and by Andrew and Johnson [27]. The corrections concern the signs and a pre-factor number. Actually, the formula needs clarification, as pointed out by Feng et al. [28]. This confusion still exists nowadays; many papers report Pabst's formula, sometimes only citing Klassen-Nejkyudova's review book [29], whereas the 
correct correspondence formula is that given Andrew and Johnson. In matrix form, the correspondence is:

$$
\mathbf{C}^{\alpha \rightarrow \gamma}=\mathbf{I}-\frac{2}{\mathbf{p}^{\mathrm{t}} \cdot \mathbf{w}}\left(\mathbf{w} \cdot \mathbf{p}^{\mathrm{t}}\right)
$$

Any reticular vector $\mathbf{r}_{\gamma}$ written in the basis $\mathfrak{B}_{c}^{\gamma}$ becomes after distortion a reticular vector of the twin written in the basis $\boldsymbol{B}_{c}^{\alpha}$ by $\mathbf{r}_{\alpha}=\mathbf{T}^{\alpha \rightarrow \gamma} \mathbf{F}^{\gamma} \mathbf{r}_{\gamma}^{\prime}=\mathbf{C}^{\alpha \rightarrow \gamma} \mathbf{r}_{\gamma}^{\prime}$. It can be easily checked that for any vector $\mathbf{r}$ in the plane $\mathbf{p}, \mathbf{r}_{\alpha}=\mathbf{C}^{\alpha \rightarrow \gamma} \mathbf{r}_{\gamma}^{\prime}=\mathbf{r}_{\gamma}$ and that $\mathbf{C}^{\alpha \rightarrow \gamma} \mathbf{w}_{\gamma}=-\mathbf{w}_{\alpha}$. This proves that formula (10) is that of the correspondence matrix. Another demonstration will be given in Section 2.6. As the coordinate of the vectors $\mathbf{p}$ and $\mathbf{w}$ are integers or half-integers, and as $\mathbf{p}^{\mathbf{t}} \cdot \mathbf{w}=q$ is integer, $\mathbf{C}$ is necessarily rational. This is a general property of the correspondence matrices [23]. It can also be checked that $\left(\mathbf{C}^{\alpha \rightarrow \gamma}\right)^{2}=\mathbf{I}$, which also implies that $\mathbf{C}^{\gamma \rightarrow \alpha}=\left(\mathbf{C}^{\alpha \rightarrow \gamma}\right)^{-1}=\mathbf{C}^{\alpha \rightarrow \gamma}$. With the convention that twinning transforms a right-hand basis into a left-hand one, $\operatorname{Det}(\mathbf{C})=-1$.

Bevis and Crocker [14] proposed an interesting formula to calculate the shear value $s$ from the correspondence matrix. The demonstration is based on the fact that a rotation or a mirror symmetry $\mathbf{T}$ preserves the metrics, and consequently the metric tensor $\mathcal{M}$ verifies the property $\mathrm{T}^{\mathrm{t}} \mathcal{M T}=\mathcal{M}$. As the correspondence matrix $\mathrm{C}$ is linked to the orientation matrix $\mathrm{T}$ and distortion matrix $\mathbf{F}$, by $\mathbf{C}=\mathbf{T F}$, we get $\mathbf{C}^{\mathrm{t}} \mathbf{M C}=\mathbf{F}^{\mathrm{t}} \mathbf{T}^{\mathrm{t}} \mathbf{M T F}=\mathbf{F}^{\mathrm{t}} \mathbf{M F}$. Using Equation (5), it leads to $\mathbf{C}^{\mathrm{t}} \boldsymbol{M C}=\boldsymbol{M}+s\left(\boldsymbol{M} \tilde{\mathbf{d}} \cdot \tilde{\mathbf{p}}^{\mathrm{t}}+\tilde{\mathbf{p}} \cdot \tilde{\mathbf{d}}^{\mathrm{t}} \boldsymbol{M}\right)+s^{2} \tilde{\mathbf{p}} \cdot \tilde{\mathbf{d}}^{\mathrm{t}} \boldsymbol{M} \tilde{\mathbf{d}} \cdot \tilde{\mathbf{p}}^{\mathrm{t}}$. By multiplying both left sides of the equality by $\mathcal{M}^{-1}$, and by taking the trace $\operatorname{Tr}$ and applying the general property $\operatorname{Tr}(\mathbf{A} \cdot \mathbf{B})=\operatorname{Tr}(\mathbf{B} \cdot \mathbf{A})$, we get $\operatorname{Tr}\left(\mathcal{M}^{-1} \mathbf{C}^{\mathrm{t}} \mathbf{M C}\right)=3+s^{2}$, which leads to Bevis and Crocker's formula:

$$
s=\sqrt{\operatorname{Tr}\left(\mathcal{M}^{-1} \mathbf{C}^{\mathrm{t}} \mathbf{M C}\right)-3}
$$

Using similar arguments, the shear value $s$ can be extracted from the shear matrix $\mathbf{F}$ by:

$$
s=\sqrt{\operatorname{Tr}\left[\mathbf{M}(\mathbf{F}-\mathbf{I}) \mathcal{M}^{-1}(\mathbf{F}-\mathbf{I})^{\mathrm{t}}\right]}
$$

\subsection{The Conjugate Type II Twins Deduced from the Type I Twins}

In the literature, the twins are given by the four elements $\mathbf{K}_{1}, \boldsymbol{\eta}_{1}, \mathbf{K}_{2}, \boldsymbol{\eta}_{2}$ without detailed explanations on their calculation. From our understanding, the type I twins are first calculated by choosing a rational plane $\mathbf{p}=\mathbf{K}_{1}$, and by defining a rational vector $\mathbf{w}=\eta_{2}$ as close as possible to the normal $\mathbf{n}$ of the plane $\mathbf{p}$. The vectors $\mathbf{w}$ in the first layer $\left(\mathbf{p}^{\mathbf{t}} \cdot \mathbf{w}=q=1\right.$, primitive cell) and then the vectors $\mathbf{w}$ of the higher levels $q$ are investigated. A threshold limit must be chosen for $q$. From $\mathbf{p}$ and $\mathbf{w}$, the shear direction $\mathbf{d} / / \eta_{1}$ is calculated with Equation (6). Then, the second plane $\mathbf{K}_{2}$ is determined as the plane that contains simultaneously the direction $\eta_{2}$ and the normal to the plane $\mathbf{K}$. The plane $\mathbf{K}$ is the plane that contains the direction $\eta_{1}$ and $\eta_{2}$. It is represented by the circle in the $(\mathbf{x}, \mathbf{y})$ plane that is transformed into the ellipse in Figure 1a. It is given by the reciprocal vector $\mathbf{K}=\eta_{1} \wedge \eta_{2}$. The plane $\mathbf{K}$ is sometimes called "plane of shear" [10], but to our opinion this term should be avoided because of its confusion with the shear plane $\mathbf{p}$. We propose to call $\mathbf{K}$ the "globally invariant normal plane" as it will be justified in Section 2.7. The plane $\mathbf{K}_{2}$ is a reciprocal vector given by $\mathbf{K}_{2}=\boldsymbol{\eta}_{1} \wedge \mathcal{M}^{*} \mathbf{K}$. With our notations it is:

$$
\mathbf{K}_{2}=\mathbf{d} \wedge\left(\mathcal{M}^{*}(\mathbf{d} \wedge \mathbf{w})\right)
$$

Now, we can build the conjugate twin of the type I twin by considering the shear plane $\mathbf{p}=\mathbf{K}_{2}$ and the shear direction $\mathbf{d} / / \eta_{2}$. As $\eta_{2}$ is rational and $\mathbf{K}_{2}$ is not necessarily rational, the conjugate twin is of type II. The macroscopic interface or habit plane between the parent crystal and its twin is the plane $\mathbf{K}_{2}$, or a rational plane close to $\mathbf{K}_{2}$. The interface is probably decomposed into rational ledges and terraces at the microscopic scale. 
In summary, the type I twins are defined from two inputs: the rational plane $\mathbf{p}=\mathbf{K}_{1}$ and the rational direction $\mathbf{w}=\eta_{2}$. The vector $\mathbf{w}$ could be actually deduced from $\mathbf{p}$ and $q$. The outputs are the shear direction $\mathbf{d} / / \boldsymbol{\eta}_{1}$ (not necessarily rational) and the rotated plane $\mathbf{K}_{2}$ (not necessarily rational). The conjugate type II twins are then defined from the shear plane $\mathbf{p}=\mathbf{K}_{2}$ (not necessarily rational) and the rational shear direction $\mathbf{d} / / \boldsymbol{\eta}_{2}$, as synthetized in Table 1 .

Table 1. Twinning elements for a twin and its conjugate. The plane $\mathbf{K}_{1}$ and the direction $\boldsymbol{\eta}_{2}$ are rational. The vectors $\mathbf{p}$ and $\mathbf{d}$ are the shear plane and shear direction, respectively. A sign "=" or "//" is added to specify the relation between the vectors.

\begin{tabular}{cccccc}
\hline & Type & $\mathbf{K}_{\mathbf{1}}$ & $\mathbf{K}_{\mathbf{2}}$ & $\boldsymbol{\eta}_{\mathbf{1}}$ & $\boldsymbol{\eta}_{\mathbf{2}}$ \\
\hline Twin & I & $\mathbf{p}=$ & rotated & $\mathbf{d} / /$ & $\mathbf{w}=$ \\
Conjugate & II & rotated & $\mathbf{p}=$ & $\mathbf{w}=$ & $\mathbf{d} / /$ \\
\hline
\end{tabular}

The shear amplitude is the same for a twin and its conjugate. This can be demonstrated by considering the globally invariant normal plane $\mathbf{K}$ in Figure 4 . It is constituted of the vectors $\eta_{1}$ and $\eta_{2}$. The planes $\mathbf{K}_{1}$ and $\mathbf{K}_{2}$ are viewed on their edges. Simple geometry arguments shows that the obliquity between the direction $\boldsymbol{\eta}_{2}$ and the normal to the plane $\mathbf{K}_{1}$ is the same as the obliquity between the direction $\boldsymbol{\eta}_{1}$ and the normal to the plane $\mathbf{K}_{2}$. This is the angle $\theta$ noted in the figure. Since $s=2$ $\tan (\theta)$, the shear is the same for a twin and for its conjugate. Another demonstration consists in noting that $\left|\eta_{1} \cdot \eta_{2}\right|=\frac{\left\|\mathbf{d}_{1}\right\|}{2}\left\|\boldsymbol{\eta}_{1}\right\|=\frac{\left\|\mathbf{d}_{2}\right\|}{2}\left\|\boldsymbol{\eta}_{2}\right\|$, and that $h_{1}\left\|\boldsymbol{\eta}_{1}\right\|=h_{2}\left\|\boldsymbol{\eta}_{2}\right\|$ because it is area of the rhombus $\mathbf{K}$. Consequently, $s_{1}=\frac{\left\|\mathbf{d}_{1}\right\|}{h_{1}}=\frac{\left\|\mathbf{d}_{2}\right\|}{h_{2}}=s_{2}$.
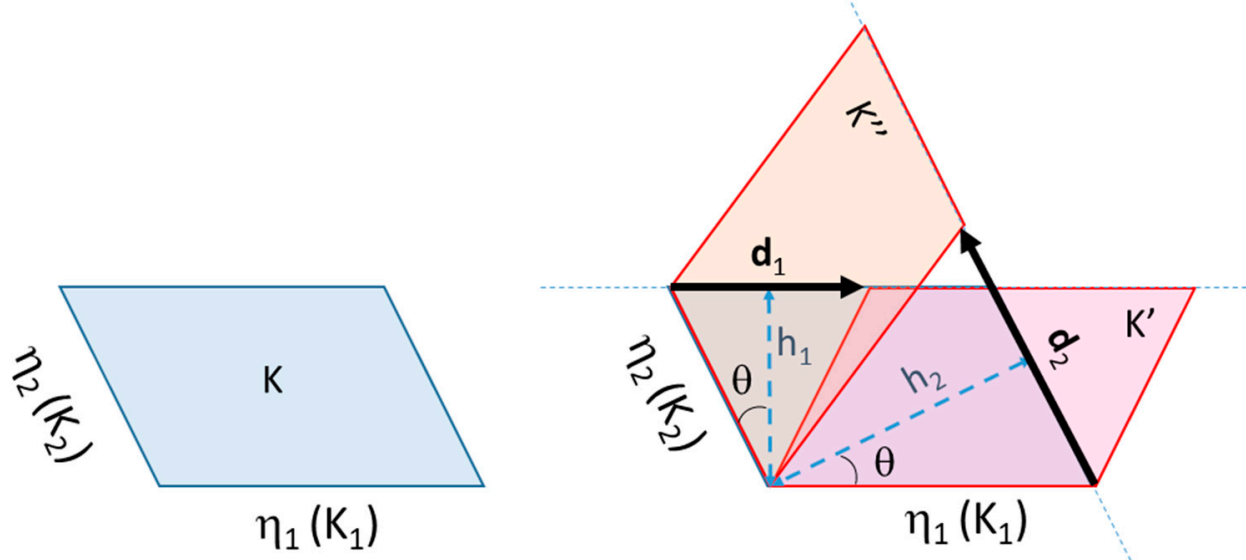

Figure 4. Scheme showing a flat view of the $\mathbf{K}$ plane and explaining why the shear value is the same for a twin and its conjugate: the shear vectors are noted $\mathbf{d}_{1}$ and $\mathbf{d}_{2}$, respectively, and the plane $\mathbf{K}$ becomes $\mathbf{K}^{\prime}$ and $\mathbf{K}^{\prime \prime}$, respectively. The obliquity $\theta$ is the same. The planes $\mathbf{K}_{1}$ and $\mathbf{K}_{2}$ are viewed on their edges.

A 2D example with a rectangular crystal twinned along its diagonal is shown in Figure 5. Please note that the conjugate twin is actually a case forbidden by the usual theory because the twin plane is a mirror symmetry and because the shear vector is rational, but we think that the theory should evolve to include this case, as it will discussed in Section 6.4. 

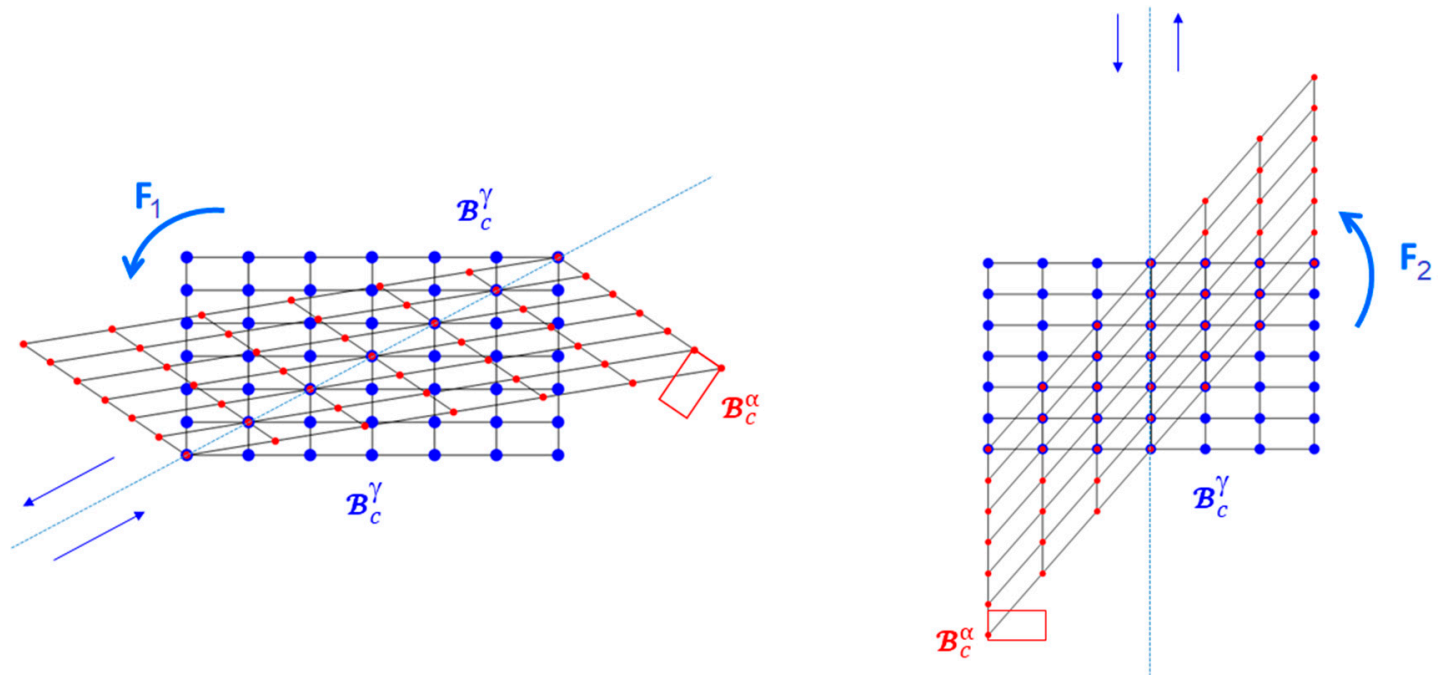

Figure 5. Conjugate twins. The $2 \mathrm{D}$ rectangular twins are defined by $\mathbf{K}_{1}=(-1,1), \boldsymbol{\eta}_{1}=-[1,1], \mathbf{K}_{2}=(1$, $0), \eta_{2}=[0,1]$ (details given in Appendix A); the direct twin $\mathbf{F}_{1}$ is on the left-hand side and its conjugate twin $\mathbf{F}_{2}$ on the right-hand side. The twin $\mathbf{F}_{1}$ is calculated with $\mathbf{p}=\mathbf{K}_{1}, \mathbf{w}=\boldsymbol{\eta}_{2}$, and its conjugate $\mathbf{F}_{2}$ with $\mathbf{p}=\mathbf{K}_{2}$ and $\mathbf{d} / / \boldsymbol{\eta}_{2}$.

The method we described to determine $\boldsymbol{\eta}_{1}$ and $\mathbf{K}_{2}$ from $\mathbf{K}_{1}$ and $\boldsymbol{\eta}_{2}$ is used to determine the type II twins from type I twins. However, to our knowledge, it has never been explained that the type II twins can also be determined independently of the type I twins. We will show in Section 5 that this is possible by conceptualizing the type II twins as real reciprocal twins, i.e., twins of the reciprocal lattice. For this purpose, we need to introduce some mathematics to write matrices in the reciprocal space.

\subsection{The Transformation Matrices Expressed in the Reciprocal Space}

First, we introduce the general notions of "full invariance" and "global invariance" for a plane. A plane $g$ of the direct space is "fully invariant" by the distortion $\mathbf{F}$ if all the directions $\mathbf{r}$ it contains are invariant by $\mathbf{F}$, i.e., $\left(\forall \mathbf{r}, g^{t} \cdot \mathbf{r}=0\right) \Rightarrow(\mathbf{F r}=\mathbf{r})$. A plane $g$ of the direct space will be said "globally invariant" by a distortion $\mathbf{F}$ if $\mathbf{F}^{*} \boldsymbol{g}=\boldsymbol{g}$, with $\mathbf{F}^{*}=\mathbf{F}^{-\mathrm{t}}$. A plane that is globally invariant is unrotated and its inter-reticular distance is preserved, but some of the directions it contains can be rotated or distorted (even if they remains in the plane). In type I twins, the plane $\mathbf{K}=\boldsymbol{\eta}_{1} \wedge \boldsymbol{\eta}_{2}$ is globally invariant but not fully invariant. As $\mathbf{K}$ is perpendicular to the shear plane $\mathbf{K}_{1}$, we call it "the globally invariant normal" plane. Actually, all the planes that contains the direction $\mathbf{d}$ are globally invariant, and only $\mathbf{p}$ is fully invariant.

Now, let us consider again the shear matrix $\mathbf{F}$ expressed in the direct space by the Equation (5). Any direction $\mathbf{u}$ of the lattice is transformed into a new direction $\mathbf{u}^{\prime}=\mathbf{F} \mathbf{u}$. The same shear acts in the reciprocal space, and the action is expressed with $\mathbf{F}^{*}=\mathbf{F}^{-\mathrm{t}}$. Any plane $\boldsymbol{h}$ of the lattice is transformed into a new plane by $\boldsymbol{h}^{\prime}=\mathbf{F}^{*} \boldsymbol{h}$. It can be shown that:

$$
\mathbf{F}^{*}=\mathbf{I}-s\left(\tilde{\mathbf{p}} \cdot \tilde{\mathbf{d}}^{t}\right)
$$

In other words, a shear on the plane $\mathbf{p}$ along the direction $\mathbf{d}$ with an amplitude $s$ in the direct space is also a shear on the plane $\mathbf{d}$ along the direction $\mathbf{p}$ with an amplitude $s^{*}=-s$ in the reciprocal space, as illustrated in Figure 6. Please note that switching from direct space to reciprocal space induces a switch of the vectors $\mathbf{d}$ and $\mathbf{p}$ with their associated metrics. Let us explain why $\mathbf{d}$ is the shear plane of the reciprocal lattice. In the direct space, the direction $\mathbf{d}$ is common to all the planes $g$ that check the condition $\mathbf{d}^{t} \cdot g=0$, i.e., all the planes $g$ that are in "zone axis" with the direction $\mathbf{d}$. Among them, there are the plane $\mathbf{p}$ which is left fully invariant by $\mathbf{F}$ and the plane $\mathbf{K}$ introduced in Section 2.6 which 
is globally invariant. For the directions, whatever the space, the nature of invariance (full or global) does not matter; they are simply invariant. In the reciprocal space, the planes $g$ become the directions $g$, and the zone axis direction $\mathbf{d}$ becomes the plane $\mathbf{d}$ that contains the directions $g$. All the reciprocal directions $g$ belonging the reciprocal plane $\mathbf{d}$ are invariant by the shear; consequently, the $\mathbf{d}$-plane is fully invariant.

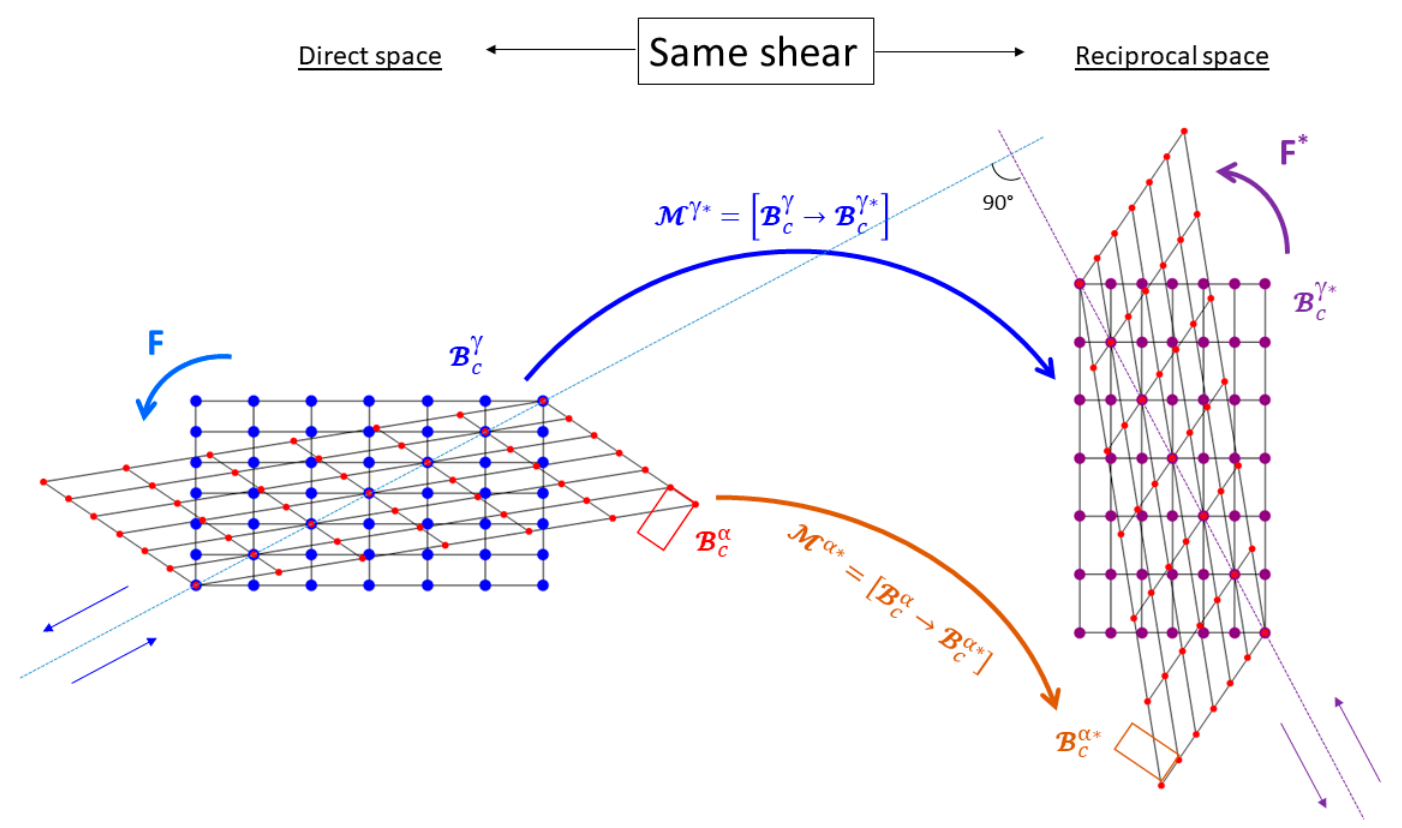

Figure 6. Twinning in the reciprocal space. The $2 \mathrm{D}$ rectangular twin defined by $\mathbf{K}_{1}=(-1,1), \boldsymbol{\eta}_{1}=-(1$, $1), \mathbf{K}_{2}=(1,0), \eta_{2}=[0,1]$ (Appendix A) is viewed in the direct space on the left-hand side, and in the reciprocal space on the right-hand side. $\mathbf{F}$ and $\mathbf{F}^{*}$ designate the same shear action; only the space in which they are illustrated changes.

A type I twin is defined by $\mathbf{p}$ rational and $\mathbf{d}$ not necessarily rational, and its conjugate type II twin by $\mathbf{d}$ rational and $\mathbf{p}$ not necessarily rational. As working in the reciprocal space reverses the planes and directions, type II twins in the direct space are type I twins in the reciprocal space, and type I twins in the direct space are type II twins in the reciprocal space. However, we point out that the transformation matrices of the type II twins in the direct space (i.e., the usual type II twins of literature) cannot be defined just by exchanging $\mathbf{d}$ and $\mathbf{p}$, or by taking the inverse of the transpose of the matrices of the type I twins in the direct space. Actually, it will be shown in Section 5 that the d-planes should be used to split the reciprocal lattice into layers in order to determine type II twins, exactly as it was done to determine the type I twins from the $\mathbf{p}$-planes of the direct lattice.

If $\mathbf{T}$ is the orientation matrix of the twin written in the direct space by Equation (8), it becomes in the reciprocal space $\mathbf{T}^{*}=\mathbf{T}^{-\mathrm{t}}$; the calculations show that:

$$
\mathbf{T}^{*}=m_{(\mathbf{n})}^{*}=\mathbf{I}-2\left(\tilde{\mathbf{p}} \cdot \mathbf{n}^{\mathbf{t}}\right)
$$

which is a mirror symmetry through the plane $\mathbf{n}$. Indeed $\mathbf{T}^{*} \tilde{\mathbf{p}}=-\tilde{\mathbf{p}}$, and for all the directions $\mathbf{g}$ of the reciprocal space such that $\mathbf{n}^{\mathbf{t}} \mathbf{g}=0$ (i.e., the planes $\mathbf{g}$ in the direct space that contain the direction $\mathbf{n}$ ), then $\mathbf{T}^{*} \mathbf{g}=\mathbf{g}$. This means that the planes containing the normal direction $\mathbf{n}$ are indexed similarly in the parent and twin crystals.

If $\mathbf{C}$ is the correspondence matrix of the twin written in the direct space by Equation (10), it becomes in the reciprocal space $\mathbf{C}^{*}=\mathbf{C}^{-\mathbf{t}}$. There is no general dyadic expression made of $\mathbf{p}$ and $\mathbf{w}$ for $\mathbf{C}^{*}$. A general expression similar to Equation (10) can be determined only by considering twinning as a shear on a rational plane of the reciprocal lattice, as it will be done in Section 5. 


\section{Direct Determination of the Transformation Matrices with the Help of Supercell Matrices}

The transformation matrices that were presented in the previous sections have been determined and reported along the last 130 years. The method to obtain them results from a mixture of geometrical considerations and intuitions, which sometimes led to confusions, as that reported by Feng et al. [28]. There is however a direct and unambiguous way to find the formulae of $\mathbf{F}, \mathbf{T}$ and $\mathbf{C}$. It is based on the explicit formulation of the matrices that define the supercell associated with the twin, as introduced in Reference [23]. Let us write $\mathfrak{B}_{\text {super }}^{\gamma}=\left(\mathbf{u}_{\gamma}, \mathbf{v}_{\gamma}, \mathbf{w}_{\gamma}\right)$ the basis of the supercell formed by the vectors of the parent crystal, $\mathfrak{B}_{\text {super }}^{\gamma \prime}=\left(\mathbf{u}_{\gamma}, \mathbf{v}_{\gamma}, \mathbf{w}_{\gamma}^{\prime}\right)$ the basis of the distorted supercell, and $\mathfrak{B}_{\text {super }}^{\alpha}=\left(\mathbf{u}_{\alpha}, \mathbf{v}_{\alpha},-\mathbf{w}_{\alpha}\right)$ the basis of the same supercell formed by the vectors of the twin. The matrices that correspond to these supercells are $\mathbf{B}_{\text {super }}^{\gamma}=\left[\mathbf{u}_{\gamma}, \mathbf{v}_{\gamma}, \mathbf{w}_{\gamma}\right]_{/ \mathfrak{B}_{c}^{\gamma}} \mathbf{B}_{\text {super }}^{\gamma \prime}=\left[\mathbf{u}_{\gamma}, \mathbf{v}_{\gamma}, \mathbf{w}_{\gamma}^{\prime}\right]_{/ \mathfrak{B}_{c}^{\gamma}}$ and $\mathbf{B}_{\text {super }}^{\alpha}=\left[\mathbf{u}_{\alpha}, \mathbf{v}_{\alpha},-\mathbf{w}_{\alpha}\right]_{/ \mathfrak{B}_{c}^{\alpha}}$; they are obtained by writing the coordinates of the vectors in column in their respective crystallographic bases. As the coordinates of the vectors $\mathbf{u}_{\alpha}, \mathbf{v}_{\alpha}, \mathbf{w}_{\alpha}$ in $\mathfrak{B}_{c}^{\alpha}$ are the same as those of $\mathbf{u}_{\gamma}, \mathbf{v}_{\gamma}, \mathbf{w}_{\gamma}$ in $\mathfrak{B}_{c}^{\gamma}$, we get $\left[\mathbf{u}_{\gamma}, \mathbf{v}_{\gamma}, \mathbf{w}_{\gamma}\right]_{/ \mathfrak{B}_{c}^{\gamma}}=\left[\mathbf{u}_{\alpha}, \mathbf{v}_{\alpha}, \mathbf{w}_{\alpha}\right]_{/ \mathfrak{B}_{c}^{\alpha}}$, i.e., the two tables of numbers are the same. Similarly, $\mathbf{B}_{\text {super }}^{\alpha}=\left[\mathbf{u}_{\alpha}, \mathbf{v}_{\alpha},-\mathbf{w}_{\alpha}\right]_{/ \mathfrak{B}_{c}^{\alpha}}=\left[\mathbf{u}_{\gamma}, \mathbf{v}_{\gamma},-\mathbf{w}_{\gamma}\right]_{/ \mathfrak{B}_{c}^{\gamma}}$. Consequently, the three supercell matrices can be expressed by tables of three column-vectors expressed in the basis $\mathfrak{B}_{c}^{\gamma}$ (the index $/ \mathfrak{B}_{c}^{\gamma}$ will be omitted to gain visibility):

$$
\begin{aligned}
& \mathbf{B}_{\text {super }}^{\gamma}=\left[\mathbf{u}_{\gamma}, \mathbf{v}_{\gamma}, \mathbf{w}_{\gamma}\right] \\
& \mathbf{B}_{\text {super }}^{\gamma \prime}=\left[\mathbf{u}_{\gamma}, \mathbf{v}_{\gamma}, \mathbf{w}_{\gamma}^{\prime}\right] \\
& \mathbf{B}_{\text {super }}^{\alpha}=\left[\mathbf{u}_{\gamma}, \mathbf{v}_{\gamma},-\mathbf{w}_{\gamma}\right]
\end{aligned}
$$

with $\mathbf{w}_{\gamma}^{\prime}=\mathbf{w}_{\gamma}+\mathbf{d}_{\gamma}$. Now, the transformation matrices according [23] are simply:

$$
\begin{aligned}
& \mathbf{F}^{\gamma}=\mathbf{B}_{\text {super }}^{\gamma \prime} \cdot\left(\mathbf{B}_{\text {super }}^{\gamma \prime}\right)^{-1} \\
& \mathbf{T}^{\alpha \rightarrow \gamma}=\mathbf{B}_{\text {super }}^{\alpha} \cdot\left(\mathbf{B}_{\text {super }}^{\gamma \prime}\right)^{-1} \\
& \mathbf{C}^{\alpha \rightarrow \gamma}=\mathbf{B}_{\text {super }}^{\alpha} \cdot\left(\mathbf{B}_{\text {super }}^{\gamma}\right)^{-1}
\end{aligned}
$$

It can be checked that $\mathbf{C}^{\alpha \rightarrow \gamma}=\mathbf{T}^{\alpha \rightarrow \gamma} \mathbf{F}^{\gamma}$, as expected. These products have similar characteristics; they are all of type $[\mathbf{u}, \mathbf{v}, \mathbf{y}] \cdot[\mathbf{u}, \mathbf{v}, \mathbf{x}]^{-1}$. Calculation show that a general formula exists for this form:

$$
[\mathbf{u}, \mathbf{v}, \mathbf{y}] \cdot[\mathbf{u}, \mathbf{v}, \mathbf{x}]^{-1}=\mathbf{I}+\frac{1}{\mathbf{p}^{\mathrm{t}} \cdot \mathbf{x}}\left((\mathbf{y}-\mathbf{x}) \cdot \mathbf{p}^{\mathrm{t}}\right)
$$

with $\mathbf{p}=(\mathbf{u} \wedge \mathbf{v})$ the cross-product of $\mathbf{u}$ by $\mathbf{v}$. The vector $\mathbf{p}$ is a direction in the reciprocal space; it is the plane $(\mathbf{u}, \mathbf{v})$ in the direct space.

The distortion matrix $\mathbf{F}^{\gamma}$ is obtained with $\mathbf{y}=\mathbf{w}_{\gamma}^{\prime}$ and $\mathbf{x}=\mathbf{w}_{\gamma}$. Since $\mathbf{y}-\mathbf{x}=\mathbf{w}_{\gamma}^{\prime}-\mathbf{w}_{\gamma}=\mathbf{d}$, it comes immediately that:

$$
\mathbf{F}^{\gamma}=\mathbf{I}+\frac{1}{\mathbf{p}^{\mathrm{t}} \cdot \mathbf{w}}\left(\mathbf{d} \cdot \mathbf{p}^{\mathrm{t}}\right)
$$

Comparing it with Equation (5) leads to the shear value $s=\frac{\|\mathbf{d}\| \cdot\|\mathbf{p}\|^{*}}{\mathbf{p}^{\mathrm{t}} \cdot \mathbf{w}}=\frac{\|\mathbf{d}\|}{q \cdot d_{h k l}}=\frac{\|\mathbf{d}\|}{h}$ which is the expected form of the shear.

The orientation matrix $\mathbf{T}^{\alpha \rightarrow \gamma}$ is obtained with $\mathbf{y}=-\mathbf{w}_{\gamma}$ and $\mathbf{x}=\mathbf{w}_{\gamma}^{\prime}$. Since $\mathbf{y}-\mathbf{x}=-\left(\mathbf{w}_{\gamma}+\mathbf{w}_{\gamma}^{\prime}\right)=-2 h \mathbf{n}$, Equation (8) derives immediately. The correspondence matrix $\mathbf{C}^{\alpha \rightarrow \gamma}$ is obtained with $\mathbf{y}=-\mathbf{w}_{\gamma}$ and $\mathbf{x}=\mathbf{w}_{\gamma}$. Since $\mathbf{y}-\mathbf{x}=-2 \mathbf{w}_{\gamma}$, Equation (10) results immediately. 


\section{The Tilted Mode}

\subsection{Specificity of the Normal Mode}

The crystallography of twins is based on the single shear paradigm. We know that twinning is not the result of a simple shear mechanism because of the steric effect of the atoms (see Section 6.4), but that is true that simple shear is a convenient and effective hypothesis to work with lattices. Even though we decide to stay inside this framework, we realized that there is an additional hypothesis that has never been explicitly stated, probably because it seemed so obvious to the pioneers that they did not feel important to specify it. The classical theory implicitly states that the shear displacement for the deformation twins (or the misfit for the growth twins) should be calculated between the two vectors $\mathbf{w}$ and $\mathbf{w}^{\prime}$ (linked by a $180^{\circ}$ rotation around the normal $\mathbf{n}$ of the twin plane), i.e., that $\mathbf{d}=\mathbf{w}^{\prime}-\mathbf{w}$. The reason for this hypothesis dates from the earliest research on twinning. The first studies were devoted to minerals, and more specifically to structures that slightly deviate from simpler cubic ones. In these cases, the normal $\mathbf{n}$ to the plane $\mathbf{p}$ has no rational indices, because of the metrics, but it is close to a rational direction. For instance, in a monoclinic structure with lattice parameters $a \approx b \approx c$ and $\beta \approx 90^{\circ}$, the normal $\mathbf{n}$ to a plane $\mathbf{p}=(h, k, l)$ is quite close to the direction $\mathbf{r}=[h, k, l]$. In the 2D example of Figure $1 \mathbf{b}$, the normal $\mathbf{n}$ to the plane $\mathbf{p}=(0,1)$ is quite close to the direction $\mathbf{b}=[0,1]$. More generally, the deviation between the normal to the twin plane and the closest rational direction is measured by the obliquity angle $\theta$, as illustrated in Figure 2. As the obliquity $\theta=(\mathbf{w}, \mathbf{n})=\left(\mathbf{w}^{\prime}, \mathbf{n}\right)$ is defined in reference to the normal $\mathbf{n}$, we call the associated twin mode "normal". This is the only mode that has been considered by the classical theory until now. Since in many twinned minerals the obliquity is small, the amplitude $\|\mathbf{d}\|=2 h \tan (\theta)$ is also small, and $\mathbf{d}$ is indeed a small translation vector between the parent and the twin lattice. However, there is no proof that there is no other translation vectors $\mathbf{d}$ of smaller amplitude that also belong to p. Actually, in metals and alloys, and in many minerals, the obliquity can be quite large, and the shear amplitude deduced from $\mathbf{d}=\mathbf{w}^{\prime}-\mathbf{w}$ (normal mode) is not anymore necessarily the smallest translation vector. There is thus another mode that can lead to the same twin orientation with smaller shear values.

\subsection{The Tilted Mode Explained in 2D}

In the normal mode " $\mathrm{N}$ ", the vector $-\mathbf{w}_{\alpha}$ is the image of $\mathbf{w}_{\gamma}$ by (i) the shear, and (ii) by the $180^{\circ}$ rotation around the normal $\mathbf{n}$. In the tilted mode, it is not the case anymore, and we have to adapt our notation to split the roles of $\mathbf{F}$ and $\mathbf{T}$. In order to make it simple, let us consider the 2D scheme of Figure 7 in which the obliquity $\theta$ is quite large. In the layer $q=1, \mathbf{w}_{\gamma}$ the parent direction closest to the normal $\mathbf{n}$ has for image the twin direction $\mathbf{w}_{\gamma}^{\prime}=-\mathbf{w}_{\alpha}$ by a $180^{\circ}$ rotation around $\mathbf{n}$. The angle $\left(\mathbf{w}_{\gamma}, \mathbf{w}_{\gamma}^{\prime}\right)=2 \theta$ is large, and consequently, the amplitude of the shear vector in the normal mode $\mathbf{d}_{\mathrm{N}(q=1)}=\mathbf{w}_{\gamma}^{\prime}-\mathbf{w}_{\gamma}$ is also large. In the tilted mode " $\mathrm{T}^{\prime}$, the direction $-\mathbf{w}_{\alpha}$ remains the orientation image of $\mathbf{w}_{\gamma}$ by the rotation the $\left(180^{\circ}, \mathbf{n}\right)$, but it is not anymore the distortion image of $\mathbf{w}_{\gamma}$. The vector $\mathbf{t}_{\gamma}=\mathbf{w}_{\gamma}+\mathbf{u}_{\gamma}$ which, as $\mathbf{w}_{\gamma}$, points to a node of the layer $q=1$, is indeed closer to $-\mathbf{w}_{\alpha}$. In this example, the amplitude of the shear vector $\mathbf{d}_{\mathrm{T}}(q=1)=-\mathbf{w}_{\alpha}-\mathbf{t}_{\gamma}$ is smaller than that of $\mathbf{d}_{\mathrm{N}(q=1)}$. Please note that for the calculation the vector $-\mathbf{w}_{\alpha}$ and $\mathbf{t}_{\gamma}$ should be expressed in the same basis, for example in $\mathfrak{B}_{c}^{\gamma}$, by writing $\mathbf{w}_{\alpha / \gamma}=\mathbf{T}^{\gamma \rightarrow \alpha} \mathbf{w}_{\alpha}$. The notion of obliquity should be adapted; we will note the normal obliquity $\theta_{\mathrm{N}}$, instead of only $\theta$, and $\theta_{\mathrm{T}}$ the tilted obliquity. 


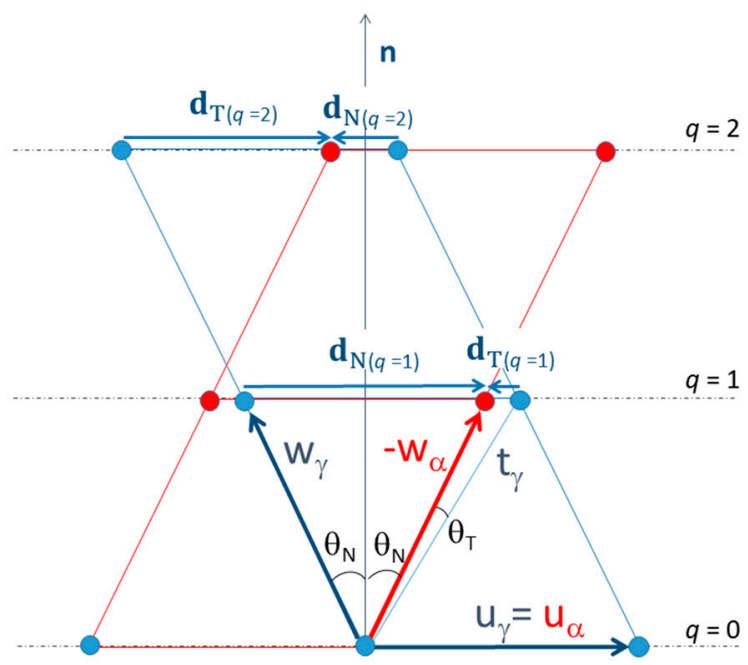

Figure 7. 2D representation of the normal and tilted modes. The parent lattice $\Upsilon$ in blue is transformed into the twin lattice $\alpha$ in red. Different shears can lead to this configuration with the same parent/twin orientation. On the layer $q=1$, the normal shear mode is represented by the vector $\mathbf{d}_{\mathrm{N}}(q=1)$, and the tilted shear mode by the vector $\mathbf{d}_{\mathrm{T}(q=1)}$. In the normal mode $-\mathbf{w}_{\alpha}$ is the image of $\mathbf{w}_{\gamma}$ by the shear. The angle $\left(\mathbf{w}_{\gamma},-\mathbf{w}_{\alpha}\right)$ is twice the obliquity, i.e., $2 \theta_{\mathrm{N}}$. In the tilted mode $-\mathbf{w}_{\alpha}$ is the image of $\mathbf{t}_{\gamma}=\mathbf{w}_{\gamma}+\mathbf{u}_{\gamma}$ by the shear. The angle $\theta_{\mathrm{T}}=\left(\mathbf{t}_{\gamma},-\mathbf{w}_{\alpha}\right)$ is the tilted obliquity.

In the example of Figure 7, the amplitude of the normal shear mode in the layer $q=1, \mathbf{d}_{\mathrm{N}}(q=1)$ is larger than that of tilted shear mode $\mathbf{d}_{\mathrm{T}}(q=1)$. If one considers the second layer $q=2$, then the amplitude $\mathbf{d}_{\mathrm{N}(q=2)}$ becomes smaller than that of $\mathbf{d}_{\mathrm{T}(q=2)}$. This situation occurs quite often in metals. Let us give some examples by comparing the literature with the results obtained by a computer program we wrote to calculate the tilted modes [25]. The $\{11 \overline{2} 1\}$ twins in magnesium are classified as $q=2$ by Crocker and Bevis [15] and by Christian and Mahajan [10], whereas Crocker and Bevis authors clearly noticed that "every point of the hexagonal Bravais lattice is sheared to its correct twin position". It seems that the authors realized this fact by geometry and not by calculation because $q=2$ signifies that only half of the lattice nodes should go to their correct position by shear. We do not understand why Crocker, Bevis, Christian and Mahajan, did not recognize the contradiction between $q=2$ and the absence of lattice shuffle. Actually, our computer program [25] shows the $\{11 \overline{2} 1\}$ twins are associated with $q=1$ in tilted mode, with the same shear value $s=0.61$ as that found by Crocker and Bevis, now without contradiction. Another example is the $\{10 \overline{1} 2\}$ extension twin in magnesium that is classified with $q=4$ by Crocker and Bevis [15] and by Christian and Mahajan [10]. Our computer program shows that these twins are actually twins with $q=2$ in a tilted mode, with the same shear value $s=0.128$. We can now specify without geometry and without any ambiguity the ratio of shuffle/shear. The primitive Bravais lattice of hexagonal compact structure is constituted of two atoms, one at the node of the lattice and an extra-one in position $(2 / 3,1 / 3,1 / 2)$, which means that twin mode with $q=1$, half of the atoms (those of the nodes) go to their correct positions by shear. The lattice shuffle is null, and the atomic shuffle is 0.5 . For the extension twins, with $q=2$, the supercell contains three extra atoms in addition to those located at the nodes of the supercell, and consequently, one quarter of the atoms go to their correct positions by shear, and three-quarter shuffle. The atomic trajectories are shown in hard-sphere model in [30]. The lattice shuffle is thus 0.5 , and the atomic shuffle is $3 / 4$. A third example is the $\{112\}$ twinning in bcc metals. The shear value $s=\frac{1}{\sqrt{2}} \approx 0.71$ is obtained by Crocker with $q=2$, even it is noticed that "no atomic shuffling is necessary". Our computer program [25] shows that $\{112\}$ twins with the same shear value are actually twins with $q=1$ in the tilted mode.

One could think from these examples, that even if the tilted mode $q=1$ is missed, it does not matter so much because the twin shear can be deduced from the normal mode with $q=2$, as in the example of Figure 7. This is not true. The tilted mode cannot always be substituted by a normal mode 
of a $q$ index twice higher. In the example of Figure 8, the tilted mode is the lowest shear mode for $q=1$ and $q=2$.

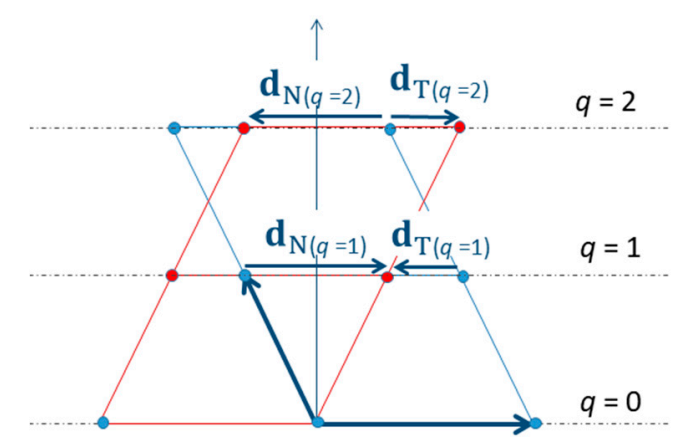

Figure 8. 2D example where the tilted mode has the lowest shear amplitude for $q=1$ and $q=2$.

The tilted mode will be said to be the "complementary" of the normal mode. A 2D example of complementary twins is shown in Figure 9.
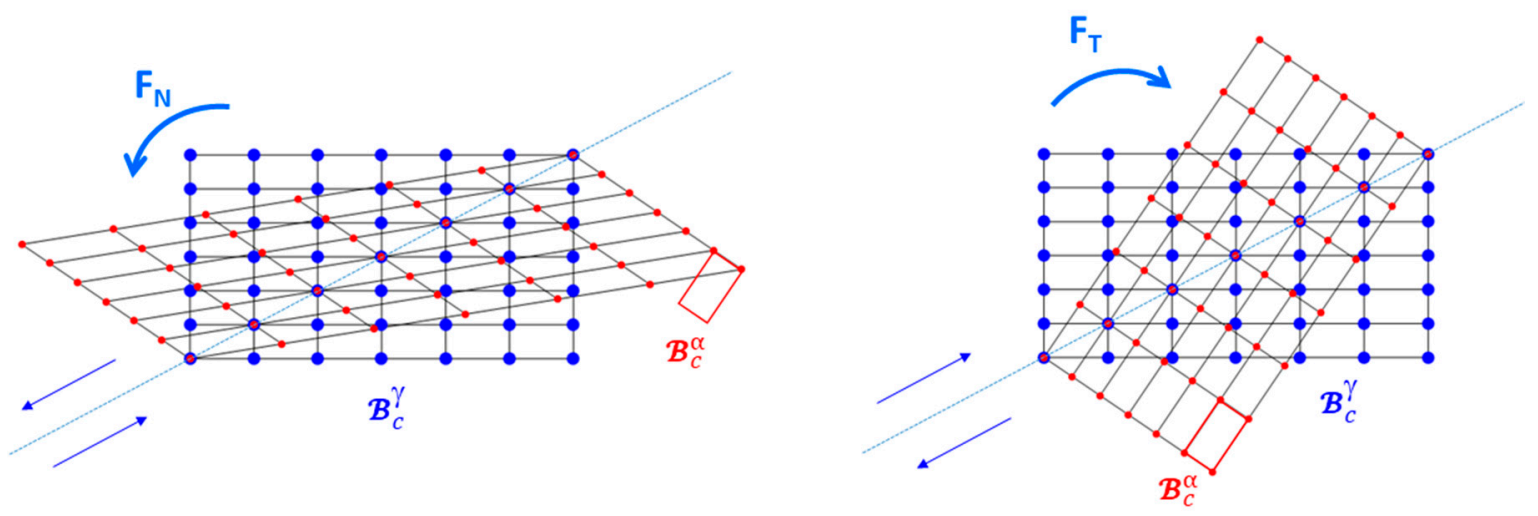

Figure 9. Complementary twins. The $2 \mathrm{D}$ rectangular twin is defined by $\mathbf{K}_{1}=(-1,1), \eta_{1}=-[1,1]$, $\mathbf{K}_{2}=(1,0), \eta_{2}=[0,1]$, and its complementary has the same elements except that the shear direction is along the opposed direction $-\eta_{1}=[1,1]$. The twinning mode is normal on the left-hand side, and tilted on the right-hand side. The shear amplitudes in both modes are calculated in Appendix A.

Let us now explain the tilted mode in 3D with Figure 10. The normal $\mathbf{n}$ intersects the $q$-layer of the plane $\mathbf{p}$ at the point $\mathrm{H}$. The four nodes of the $q$-layer "around" $\mathrm{H}$ are A, B, C and D. The node closest to $\mathrm{H}$ is $\mathrm{A}$. The image of $\mathrm{A}$ by the rotation $\left(180^{\circ}, \mathbf{n}\right)$ is the point $\mathrm{A}^{\prime}$. If the obliquity $\theta$ were very small, the node closest to $\mathrm{A}^{\prime}$ would have been A ("normal" twin mode); however, if the obliquity is large, as it is the case in the figure, the node closest to $\mathrm{A}^{\prime}$ is not anymore $\mathrm{A}$. In Figure 10, the closest node to $\mathrm{A}^{\prime}$ is $\mathrm{B}$. The twin lattice can thus be obtained by a shear along the vector $\mathbf{d}_{\mathrm{T}}=\mathrm{BA}^{\prime}$. The twinning mode is "tilted". Let us note the vector $\mathbf{t}=\mathrm{OB}$. This vector checks $\mathbf{p}^{\mathbf{t}} \cdot \mathbf{t}=\mathbf{p}^{\mathbf{t}} \cdot \mathbf{w}=q$. The vector $-\mathbf{w}_{\alpha}$, which is the image of $\mathbf{w}_{\gamma}$ by the $\mathbf{n}-180^{\circ}$ rotation, is not anymore the image of $\mathbf{w}_{\gamma}$ by the shear along $\mathbf{d}_{\mathrm{N}}$ as in the normal mode; it is the image of $\mathbf{t}_{\gamma}=\mathbf{w}_{\gamma}+\mathbf{u}_{\gamma}$ by the shear along $\mathbf{d}_{\mathrm{T}}$. 


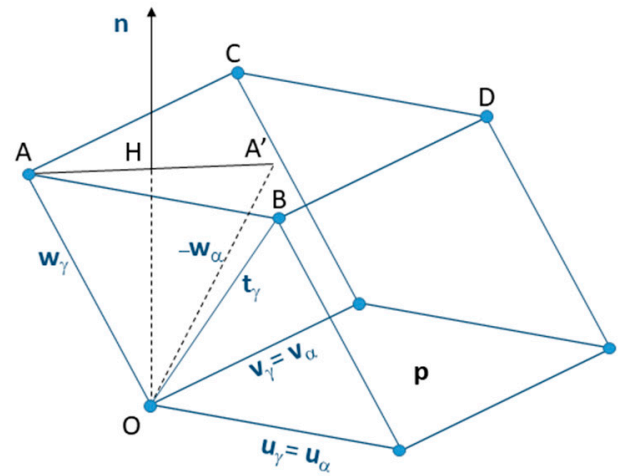

(a)

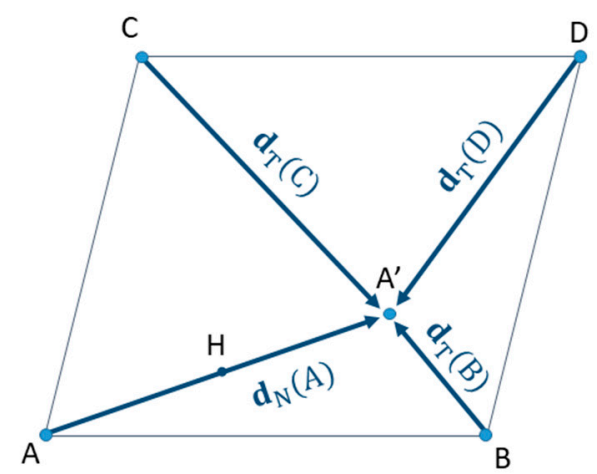

(b)

Figure 10. 3D representation of the tilted mode of twinning. (a) Supercell $(\mathbf{u}, \mathbf{v}, \mathbf{w})$. The normal $\mathbf{n}$ intersects the $q$-layer at the point $\mathrm{H}$. The four nodes of the $q$-layer "around" $\mathrm{H}$ are A, B, C and D. The point closest to $\mathrm{H}$ is $\mathrm{A}$. The image of $\mathrm{A}$ by the $\left(180^{\circ}, \mathbf{n}\right)$ rotation is $\mathrm{A}^{\prime}$. (b) Top-view of the $q$-layer. In this example, the shortest distance between $\mathrm{A}^{\prime}$ and the nodes $\mathrm{A}, \mathrm{B}, \mathrm{C}, \mathrm{D}$ is $\mathrm{BA}^{\prime}$. Thus $\mathrm{A}^{\prime}$ is the image of B by shear (and not of A as expected in a normal mode). The twinning mode is "tilted" if one assumes that the lowest shear is selected.

More generally, from this analysis, we can identify four possible shears: one normal mode (from A) and three tilted modes (from, B, C or D). The third vector $\mathbf{t}_{\gamma}$ of the sheared supercell for each of these four possibilities is given by:

$$
\begin{aligned}
& \text { Normalmode } \\
& \mathbf{t}_{\gamma}=\mathbf{w}_{\gamma} \\
& \text { Tiltedmodes } \\
& \left\{\begin{array}{l}
\mathbf{t}_{\gamma}=\mathbf{w}_{\gamma}+\mathbf{u}_{\gamma} \\
\mathbf{t}_{\gamma}=\mathbf{w}_{\gamma}+\mathbf{v}_{\gamma} \\
\mathbf{t}_{\gamma}=\mathbf{w}_{\gamma}+\mathbf{u}_{\gamma}+\mathbf{v}_{\gamma}
\end{array}\right.
\end{aligned}
$$

The shear vector $\mathbf{d}$ associated with these four possibilities are $\mathbf{d}_{\mathrm{N}}$ for the normal mode, given by Equation (6), i.e., $\mathbf{d}_{\mathrm{N}}=-2 \mathbf{w}_{\gamma}+2 h \mathbf{n}$, and $\mathbf{d}_{\mathrm{T}}=\mathbf{d}_{\mathrm{N}}-\mathbf{u}_{\gamma}, \mathbf{d}_{\mathrm{N}}-\mathbf{v}_{\gamma}$, or $\mathbf{d}_{\mathrm{N}}-\mathbf{u}_{\gamma}-\mathbf{v}_{\gamma}$ for the tilted modes. When written in the general form, they are:

$$
\mathbf{d}=-\left(\mathbf{w}_{\gamma}+\mathbf{t}_{\gamma}\right)+2 h \mathbf{n}
$$

Among the four possibilities (one normal and three tilted), the lowest shear $\|\mathbf{d}\|$ is chosen. The three tilted modes and the normal mode are said "complementary".

The transformation matrices (distortion, orientation, correspondence) can be calculated following the method described in Section 3. We write $\mathfrak{B}_{\text {superT }}^{\gamma}=\left(\mathbf{u}_{\gamma}, \mathbf{v}_{\gamma}, \mathbf{w}_{\gamma}\right)$ the basis of the supercell that is transformed into $\boldsymbol{B}_{\text {super }}^{\alpha}$ by $\mathbf{T}$ the $\mathbf{p}$-mirror symmetry, and we introduce $\boldsymbol{B}_{\text {superF }}^{\gamma}=\left(\mathbf{u}_{\gamma}, \mathbf{v}_{\gamma}, \mathbf{t}_{\gamma}\right)$ the basis of the supercell that is transformed into $\mathfrak{B}_{\text {super }}^{\alpha}$ by $\mathbf{F}$ the shear along the vector $\mathbf{d}$. The basis of the distorted supercell is $\mathfrak{B}_{\text {superF }}^{\gamma \prime}=\left(\mathbf{u}_{\gamma}, \mathbf{v}_{\gamma}, \mathbf{t}_{\gamma}^{\prime}\right)$, which is also the basis of the supercell of the twin $\mathfrak{B}_{\text {super }}^{\alpha}=\left(\mathbf{u}_{\alpha}, \mathbf{v}_{\alpha},-\mathbf{w}_{\alpha}\right)$.

Similarly as in Section 3, we define the supercell matrices. Written in the basis $\mathfrak{B}_{c}^{\gamma}$ they are:

$$
\begin{aligned}
& \mathbf{B}_{\text {super } T}^{\gamma}=\left[\mathbf{u}_{\gamma}, \mathbf{v}_{\gamma}, \mathbf{w}_{\gamma}\right] \\
& \mathbf{B}_{\text {superF }}^{\gamma}=\left[\mathbf{u}_{\gamma}, \mathbf{v}_{\gamma}, \mathbf{t}_{\gamma}\right] \\
& \mathbf{B}_{\text {superF }}^{\gamma^{\prime}}=\left[\mathbf{u}_{\gamma}, \mathbf{v}_{\gamma}, \mathbf{t}_{\gamma}^{\prime}\right] \\
& \mathbf{B}_{\text {super }}^{\alpha}=\left[\mathbf{u}_{\gamma}, \mathbf{v}_{\gamma},-\mathbf{w}_{\gamma}\right]
\end{aligned}
$$


with $\mathbf{t}_{\gamma}^{\prime}=\mathbf{t}+\mathbf{d}$. The transformation matrices are deduced from them by:

$$
\begin{aligned}
& \mathbf{F}^{\gamma}=\mathbf{B}_{\text {superF }}^{\gamma \prime} \cdot\left(\mathbf{B}_{\text {superF }}^{\gamma}\right)^{-1} \\
& \mathbf{T}^{\alpha \rightarrow \gamma}=\mathbf{B}_{\text {super }}^{\alpha} \cdot\left(\mathbf{B}_{\text {super }}^{\gamma \prime}\right)^{-1} \\
& \mathbf{C}^{\alpha \rightarrow \gamma}=\mathbf{B}_{\text {super }}^{\alpha} \cdot\left(\mathbf{B}_{\text {super }}^{\gamma}\right)^{-1}
\end{aligned}
$$

For the computation of the matrices $\mathbf{F}^{\gamma}, \mathbf{T}^{\alpha \rightarrow \gamma}$ and $\mathbf{C}^{\alpha \rightarrow \gamma}$, Equation (23) associated with Equation (20) is sufficient. However, their dyadic product forms can also be determined by using Equation (18), i.e., $[\mathbf{u}, \mathbf{v}, \mathbf{y}] \cdot[\mathbf{u}, \mathbf{v}, \mathbf{x}]^{-1}=\mathbf{I}+\frac{1}{\mathbf{p}^{\mathrm{t} \cdot \mathbf{x}}}\left((\mathbf{y}-\mathbf{x}) \cdot \mathbf{p}^{\mathrm{t}}\right)$ with $\mathbf{p}=(\mathbf{u} \wedge \mathbf{v})$ :

The distortion matrix $\mathbf{F}^{\gamma}$ is obtained with $\mathbf{y}=\mathbf{t}_{\gamma}^{\prime}$ and $\mathbf{x}=\mathbf{t}_{\gamma}$. Since $\mathbf{y}-\mathbf{x}=\mathbf{t}_{\gamma}^{\prime}-\mathbf{t}_{\gamma}=\mathbf{d}$, it comes immediately that $\mathbf{F}^{\gamma}$ is given by Equation (19), which is the expected general form of shear. However, now the shear vector $\mathbf{d}$ should be calculated with the general Equation (21).

The orientation matrix $\mathbf{T}^{\alpha \rightarrow \gamma}$ is obtained with $\mathbf{y}=-\mathbf{w}_{\gamma}$ and $\mathbf{x}=\mathbf{t}_{\gamma}^{\prime}$. Since $\mathbf{y}-\mathbf{x}=$ $-\left(\mathbf{w}_{\gamma}+\mathbf{t}_{\gamma}^{\prime}\right)=-2 h \mathbf{n}, \mathbf{T}^{\alpha \rightarrow \gamma}$ is given by Equation (8) without any change. This proves that the orientation of the twin is independent of the twinning mode (normal of tilted).

The correspondence matrix $\mathbf{C}^{\alpha \rightarrow \gamma}$ is obtained with $\mathbf{y}=-\mathbf{w}_{\gamma}$ and $\mathbf{x}=\mathbf{t}_{\gamma}$. It appears as a more general form of Equation (10):

$$
\mathbf{C}^{\alpha \rightarrow \gamma}=\mathbf{I}-\frac{1}{\mathbf{p}^{\mathrm{t}} \cdot \mathbf{w}}\left((\mathbf{w}+\mathbf{x}) \cdot \mathbf{p}^{\mathrm{t}}\right)
$$

where the reticular vector $\mathbf{t}$ is given by Equation (20).

The computer program we wrote in Python performs all these calculations in order to "predict" the twins, at least in the framework of the simple shear hypothesis. It explores all the planes $\mathbf{p}=(h$, $k, l)$ with a threshold in the indices $h, k, l$, and it calculates the four modes for each plane $\mathbf{p}$ (shear, orientation and correspondence matrices). The shear values obtained for all the explored planes $\mathbf{p}$ are then ranked from the lowest to the highest values. The results obtained for fcc, bcc, hcp metals, and for some minerals will be presented in a separated paper [25].

\section{The Type II Twins Built as Real Reciprocal Twins}

As introduced in Section 2.6, the type II twins are usually defined as "the conjugates" of type I twins. As the type I twins preserve a plane (the mirror plane) and the type II twins preserve a direction (the $180^{\circ}$ rotation axis), it was confusingly perceived that type I and type II could have a link with the direct and reciprocal spaces, respectively; but, to our knowledge, no one thought or wrote anything clear about this. We will show that type II twins are actually real reciprocal twins, i.e., they act as twins by a simple shear on a rational plane of the reciprocal space. With this idea, type II twins can be determined independently of the type I twins.

\subsection{The $q^{*}$ Layers of d-Plane in the Reciprocal Space}

For a scientist practicing transmission electron microscopy (TEM), the reciprocal space is as real as the direct space, and the switch is simply made by pressing the button "diffraction" of the microscope. More precisely, TEM diffraction allows us to see the nodes of the reciprocal lattice that are close to the Laue sphere [31]. The crystal is orientated such that the parallel electron beam is along a crystallographic direction $\mathbf{d}$ (the "zone axis"), and the diffraction spots correspond to the planes that contains this direction; they form a lattice of points called the zero-order Laue zone $q^{*}=0$, as illustrated in Figure 11. If the electron beam is not parallel, the convergent beam diffraction (CBED) allows exploring higher order Laue zones $q^{*}>0$, as shown in Figure 12. 


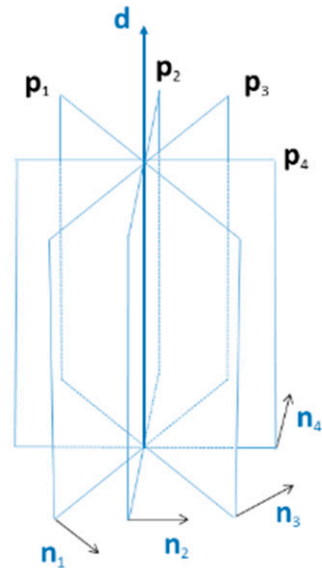

(a) Direct space

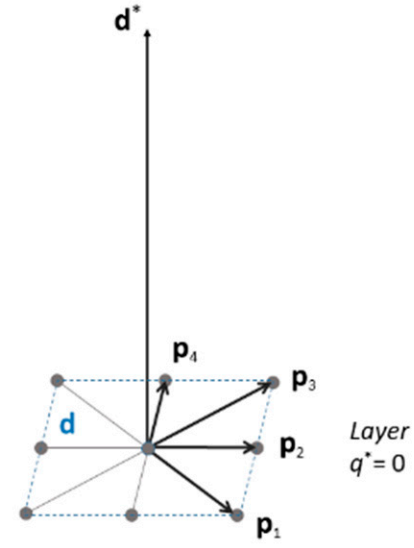

(b) Reciprocal space

Figure 11. Layer $q^{*}=0$ viewed in (a) direct space and (b) reciprocal space. In (a) the planes $\mathbf{p}$ are in zone axis with the direction $\mathbf{d}$, and in (b) they belong to the $\mathbf{d}$-plane, $\mathbf{d}^{\mathrm{t}} \cdot \mathbf{p}=0$.

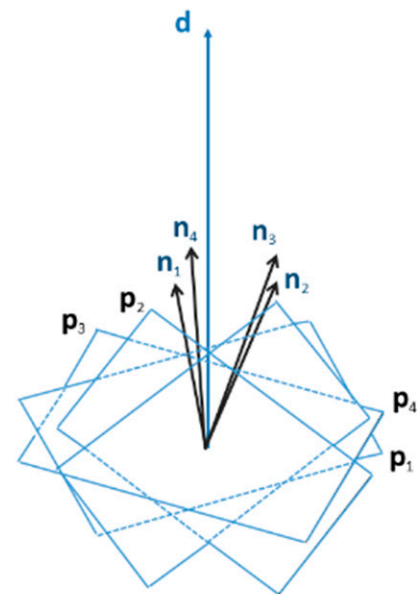

(a) Direct space

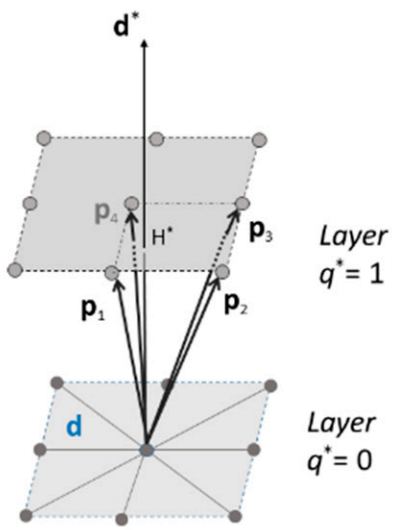

(b) Reciprocal space

Figure 12. Layer $q^{*}=1$ viewed in (a) direct space and (b) reciprocal space. The $\mathbf{d}$-plane is made of the reciprocal directions $\mathbf{p}$ such that $\mathbf{d}^{\mathrm{t}} \cdot \mathbf{p}=q^{*}$.

Similarly as the direct lattice can be imagined as layers of a $\mathbf{p}$-plane constituted of nodes $\mathbf{w}$ such that $\mathbf{p}^{\mathbf{t}} \cdot \mathbf{w}=q$, the reciprocal lattice can be imagined as layers of a $\mathbf{d}$-plane constituted by nodes $\mathbf{p}$ such that $\mathbf{d}^{\mathbf{t}} \cdot \mathbf{p}=q^{*}$. We recall that in the direct space $\mathbf{d}$ and $\mathbf{p}$ are a direction and a plane, respectively, and that in the reciprocal space they become a plane and a direction, respectively. We have also seen in Section 2.7 that type II twins in the direct space are type I twins in the reciprocal space. Let us use this property to build them.

\subsection{The Reciprocal Transformation Matrices Determined from the Reciprocal Supercell}

The type II twins can be imagined exactly as the type I twins, but now by considering a supercell $(h, k, l)$ of the reciprocal lattice instead of the supercell $(\mathbf{u}, \mathbf{v}, \mathbf{w})$ of the direct lattice, as shown in Figure 13. Here, $\boldsymbol{h}, \boldsymbol{k}, \boldsymbol{l}$ are three directions of the reciprocal space, i.e., three planes of the direct space. The vector $l$ acts as the vector $\mathbf{w}$; it defines the size of the supercell by the integer $\mathbf{d}^{\mathrm{t}} \cdot \mathbf{l}=q *$. The reciprocal length between the d-planes is $h^{*}=q^{*} / d_{u v w}$ where $d_{u v w}$ is the length of the vector $\mathbf{d}=[u, v$, $w$ ]. It is the inverse of a distance. 


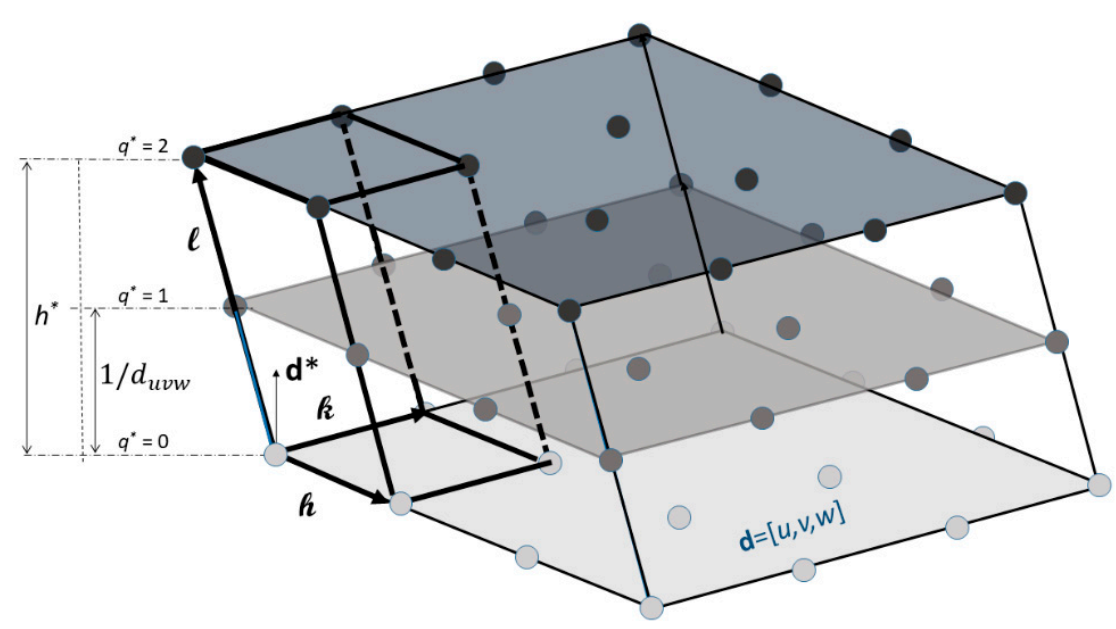

Figure 13. Supercell $(h, k, l)$ of the reciprocal lattice used to build the type II twins. Here, 1 points to a node of the second layer $q^{*}=2$. The reciprocal length between the $\mathbf{d}$-planes that are sheared is $h^{*}=q^{*} / d_{\text {uvw. }}$.

Exactly as type I twins are simple shear in the direct space, type II twins are simple shear in the reciprocal space. They are defined with the help of the supercell $\mathfrak{B}_{\text {super }}^{\gamma^{*}}=\left(\boldsymbol{h}_{\gamma}, \boldsymbol{k}_{\gamma}, \boldsymbol{l}_{\gamma}\right)$ that is sheared and becomes a supercell $\mathfrak{B}_{\text {super }}^{\alpha *}=\left(\boldsymbol{h}_{\alpha}, \boldsymbol{k}_{\alpha}, \boldsymbol{l}_{\alpha}\right)$ that is in mirror symmetry with $\mathfrak{B}_{\text {super }}^{\gamma *}$ through the reciprocal plane $\mathbf{d}=\left(\boldsymbol{h}_{\gamma}, \boldsymbol{k}_{\gamma}\right)$. As for type I twins, the type II twins can have one normal mode and three tilted modes that can be determined from the supercell, as shown in Figure 14.

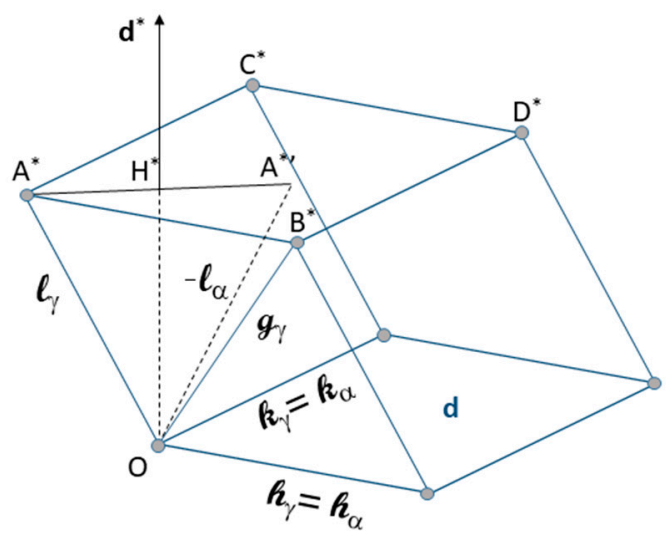

(a)

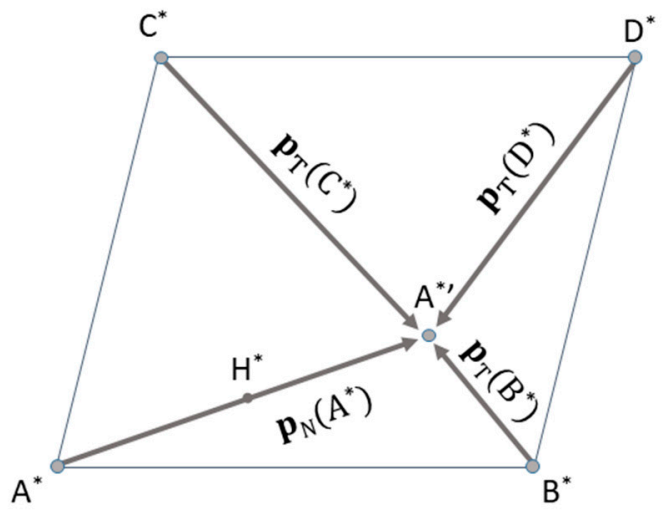

(b)

Figure 14. 3D geometrical representation in reciprocal space of a type II twin, with its normal mode (from $\mathrm{A}^{*}$ ) and its three tilted modes (from $\mathrm{B}^{*}, \mathrm{C}^{*}$ or $\mathrm{D}^{*}$ ). The figure is very similar to that of twinning in the direct space (Figure 10). (a) Supercell $(\boldsymbol{h}, \boldsymbol{k}, \boldsymbol{l})$. The normal $\mathrm{d}^{*}$ intersects the $q^{*}$-layer at the point $\mathrm{H}^{*}$. The four nodes "around" $\mathrm{H}^{*}$ are $\mathrm{A}^{*}, \mathrm{~B}^{*}, \mathrm{C}^{*}$ and $\mathrm{D}^{*}$. The point closest to $\mathrm{H}^{*}$ is $\mathrm{A}^{*}$. (b) Top-view of the $q^{*}$-layer.

The associated vector forming the third vector $g_{\gamma}$ of the supercell that is sheared is:

Normalmode

$$
\begin{aligned}
& \boldsymbol{g}_{\gamma}=\boldsymbol{l}_{\gamma} \\
& \left\{\begin{array}{l}
\boldsymbol{g}_{\gamma}=\boldsymbol{l}_{\gamma}+\boldsymbol{h}_{\gamma} \\
\boldsymbol{g}_{\gamma}=\boldsymbol{l}_{\gamma}+\boldsymbol{k}_{\gamma} \\
\boldsymbol{g}_{\gamma}=\boldsymbol{l}_{\gamma}+\boldsymbol{h}_{\gamma}+\boldsymbol{k}_{\gamma}
\end{array}\right.
\end{aligned}
$$


We define the reciprocal supercell matrices in the basis $\mathfrak{B}_{c}^{\gamma *}$ by:

$$
\begin{aligned}
& \mathbf{B}_{\text {superT }}^{\gamma^{*}}=\left[\boldsymbol{h}_{\gamma}, \boldsymbol{k}_{\gamma}, \boldsymbol{l}_{\gamma}\right] \\
& \mathbf{B}_{\text {superF }}^{\gamma *}=\left[\boldsymbol{h}_{\gamma}, \boldsymbol{k}_{\gamma}, \boldsymbol{g}_{\gamma}\right] \\
& \mathbf{B}_{\text {superF }}^{\gamma * \prime}=\left[\boldsymbol{h}_{\gamma}, \boldsymbol{k}_{\gamma}, \boldsymbol{g}_{\gamma}^{\prime}\right] \\
& \mathbf{B}_{\text {super }}^{\alpha *}=\left[\boldsymbol{h}_{\gamma}, \boldsymbol{k}_{\gamma},-\boldsymbol{l}_{\gamma}\right]
\end{aligned}
$$

with $g_{\gamma}^{\prime}=g_{\gamma}+\mathbf{p}$. The shear vector $\mathbf{p}$ associated with these four possibilities are $\mathbf{p}_{\mathrm{N}}$ for the normal mode, $\mathbf{p}_{\mathrm{N}}=-2 \boldsymbol{l}_{\gamma}+2 h^{*}(\boldsymbol{\mathcal { M }} \tilde{\mathbf{d}})$, and $\mathbf{p}_{\mathrm{T}}=\mathbf{p}_{\mathrm{N}}-\boldsymbol{h}_{\gamma}, \mathbf{p}_{\mathrm{N}}-\boldsymbol{k}_{\gamma}$, or $\mathbf{p}_{\mathrm{N}}-\boldsymbol{h}_{\gamma}-\boldsymbol{k}_{\gamma}$ for the tilted modes. They can be written in the general form:

$$
\mathbf{p}=-\left(l_{\gamma}+g_{\gamma}\right)+2 h^{*}(\mathbf{M} \tilde{\mathbf{d}})
$$

The four supercell matrices allows the calculation of the transformation matrices, which are:

$$
\begin{aligned}
& \mathbf{F}^{\gamma^{*}}=\mathbf{B}_{\text {super }}^{\gamma * \prime} \cdot\left(\mathbf{B}_{\text {superF }}^{\gamma *}\right)^{-1} \\
& \mathbf{T}^{\alpha * \rightarrow \gamma^{*}}=\mathbf{B}_{\text {super }}^{\alpha *} \cdot\left(\mathbf{B}_{\text {superF }}^{\gamma * \prime}\right)^{-1} \\
& \mathbf{C}^{\alpha * \rightarrow \gamma^{*}}=\mathbf{B}_{\text {super }}^{\alpha *} \cdot\left(\mathbf{B}_{\text {superF }}^{\gamma^{*}}\right)^{-1}
\end{aligned}
$$

The dyadic forms of these matrices is determined by using again the fact that the product $[\boldsymbol{h}, \boldsymbol{k}, \mathbf{y}] \cdot[\boldsymbol{h}, \boldsymbol{k}, \mathbf{x}]^{-1}=\mathbf{I}+\frac{1}{\mathrm{~d}^{\mathrm{t}} \cdot \mathbf{x}}\left((\mathbf{y}-\mathbf{x}) \cdot \mathbf{d}^{\mathrm{t}}\right)$ with $\mathbf{d}=(\boldsymbol{h} \wedge \boldsymbol{k})$.

The distortion matrix $\mathbf{F}^{\gamma^{*}}$ is obtained with $\mathbf{x}=g_{\gamma}$ and $\mathbf{y}=g_{\gamma}^{\prime}$. Since $\mathbf{y}-\mathbf{x}=g_{\gamma}^{\prime}-g_{\gamma}=\mathbf{p}$, it comes immediately that

$$
\mathbf{F}^{\gamma^{*}}=\mathbf{I}+\frac{1}{\mathbf{d}^{\mathrm{t}} \cdot g}\left(\mathbf{p} \cdot \mathbf{d}^{\mathrm{t}}\right)
$$

Comparing with Equation (14) and taking into account that $\mathbf{d}^{t} \cdot g=\mathbf{d}^{t} \cdot \boldsymbol{l}=q^{*}$ and $h^{*}=q^{*} / d_{\text {uvw }}$, the shear value is $s^{*}=\frac{\|\mathbf{p} \mid\|^{*}\|\mathbf{d}\|}{\mathbf{d}^{t} \cdot g}=\frac{\|\mathbf{p}\|^{*} d_{u v w}}{q^{*}}=\frac{\|\mathbf{p}\|^{*}}{h^{*}}$ which is the expected form of the shear in the reciprocal space.

The orientation matrix $\mathbf{T}^{\alpha * \rightarrow \gamma^{*}}$ is obtained with $\mathbf{y}=-g_{\gamma}$ and $\mathbf{x}=g_{\gamma}^{\prime}$. Since $\mathbf{y}-\mathbf{x}=-\left(g+g_{\gamma}^{\prime}\right)=-2 h^{*}(\boldsymbol{M} \tilde{\mathbf{d}})$, an equation similar to that of Equation (8) is deduced:

$$
\mathbf{T}^{\alpha * \rightarrow \gamma^{*}}=m_{(\mathbf{d})}^{*}=\mathbf{I}-2\left(\boldsymbol{M} \tilde{\mathbf{d}} \cdot \tilde{\mathbf{d}}^{\mathrm{t}}\right)
$$

The correspondence matrix $\mathbf{C}^{\alpha * \rightarrow \gamma^{*}}$ is obtained with $\mathbf{y}=-l_{\gamma}$ and $\mathbf{x}=g_{\gamma}$. Since $\mathbf{y}-$ $\mathbf{x}=-\left(l_{\gamma}+g_{\gamma}\right)$ an equation similar to Equation (24) is deduced:

$$
\mathbf{C}^{\alpha * \rightarrow \gamma^{*}}=\mathbf{I}-\frac{1}{\mathbf{d}^{\mathrm{t}} \cdot g}\left((\boldsymbol{l}+\boldsymbol{g}) \cdot \mathbf{d}^{\mathrm{t}}\right)
$$

with $l=l_{\gamma}$ and $g=g_{\gamma}$. For normal reciprocal twinning mode $l_{\gamma}=g_{\gamma}$, and the equation becomes:

$$
\mathbf{C}^{\alpha * \rightarrow \gamma^{*}}=\mathbf{I}-\frac{2}{\mathbf{d}^{\mathrm{t}} \cdot g}\left(g \cdot \mathbf{d}^{\mathrm{t}}\right)
$$

which is similar to Equation (10) by replacing $\mathbf{p}$ to $\mathbf{d}$ and $\mathbf{w}$ to $g$. For type II twins, by construction, $\mathbf{d}$ and $g$ are rational vectors, and consequently, $\mathbf{C}^{\alpha * \rightarrow \gamma^{*}}$ is necessarily a rational matrix. 
The three equations (30)-(32) give the transformation matrices of the type II twins in the reciprocal space. The matrices can then be simply expressed in the direct space by taking the inverse of their transposes. The computer program [25] will be used to determine the type II twins by exploring the directions $\mathbf{d}=[u, v, w]$, within a threshold in the indices $u, v, w$. The shear amplitudes will be ranked from the lowest to the highest values. This is an on-going work. Clearly, the list of twins determined from this approach is expected to be the same as that determined by calculating the conjugate twins of the type I twins. In particular, the list of shear values should be same as that of type I twins. This is not obvious if we only consider that the shear amplitudes of the type II twins associated to Equation (29) are $s^{*}=\frac{\|\mathbf{p}\|^{*}\|\mathbf{d}\|}{\mathbf{d}^{t} \cdot g}$ and those of the type I twins associated with Equation (18) are $s=\frac{\|\mathbf{d}\|\|\mathbf{p}\|^{*}}{\mathbf{p}^{\mathbf{t}} \mathbf{w}}$. It is true that both are of kind $\left\|\mathbf{d}\left|\|\mid \mathbf{p}\|^{*}\right.\right.$ divided by an integer, but $\mathbf{d}$ is rational (not necessarily $\mathbf{p}$ ) for type II twins, whereas $\mathbf{p}$ is rational (not necessarily $\mathbf{d}$ ) for the type I twins. Only the list of shear amplitudes should be the same; the list of matrices (shear, orientation and correspondence) will be different from that of the type I twins.

\section{Discussion}

\subsection{Synthesis}

We explained in details the crystallography of twinning inside the Friedelian framework in which twinning is assumed to result from a simple shear mechanism and where only the lattice (simple or multiple) and its metrics matter. The model for deformation twins and growth twins is the same; formally, there is no mathematical difference between the shear vector $\mathbf{d}$ used for the formers, and the lattice misfits used for the latter. In both cases, the model assumes that the amplitude of the shear/misfit should be small in order to facilitate the accommodation and reduce the accommodation energy at the interface and in the bulk around the twin. For type I twins, we used the convention that the parent and twin lattices are in mirror symmetry through a plane $\mathbf{p}$ (of normal $\mathbf{n}$ ). We explicitly wrote the transformation matrices (distortion $\mathbf{F}$, orientation $\mathbf{T}$ and correspondence $\mathbf{C}$ ) in dyadic matrix forms with the convention the determinant of $\mathbf{F}$ is 1 , and that of $\mathbf{T}$ and $\mathbf{C}$ is -1 . Another convention could have been chosen in which the parent and its twin are related by a $180^{\circ}$ rotation around $\mathbf{n}$. In that case, the transformation matrices $\mathbf{T}$ and $\mathbf{C}$ would have a determinant equal to 1 . We also introduced two new concepts.

The first novelty is the "tilted mode". The classical twinning model implicitly assumes that a reticular direction $\mathbf{w}$ should be "close to" the normal $\mathbf{n}$ of the twin plane $\mathbf{p}$ and that the twin is such that $\mathbf{w}$ becomes $\mathbf{w}^{\prime}$ by a small shear $\mathbf{d}$ (deformation twinning) or by a small misfit (growth twinning) with $\mathbf{w}^{\prime}-\mathbf{w}=\mathbf{d}$. In order to quantitatively estimate how $\mathbf{w}$ should be close to $\mathbf{n}$, Friedel introduced the obliquity $\theta$, which is simply the angle between $\mathbf{w}$ and $\mathbf{n}$. He found that in most of minerals, growth twins are formed with obliquities below 5 or $6^{\circ}$, which would correspond to a shear amplitude of $s$ $\sim 0.2$. However, in metallurgy, some twinning shear reported in literature can be as high as 0.7 . It does not imply that Friedel's approach cannot be applied anymore, but the hypothesis $\mathbf{d}=\mathbf{w}^{\prime}-\mathbf{w}$ should be generalized. Let us consider again Friedel's assumption: the reticular direction of twin $\mathbf{w}_{\alpha}$ and the reticular direction of the parent $\mathbf{w}_{\gamma}$ are in mirror symmetry through the plane $\mathbf{p}$ and are such that the image $-\mathbf{w}_{\alpha}=\mathbf{w}_{\gamma}^{\prime}$ where $\mathbf{w}_{\gamma}^{\prime}$ is the image of $\mathbf{w}_{\gamma}$ by the shear. In other words, Friedel assumed a correlation between the mirror orientation $\mathbf{T}$ and the lattice distortion $\mathbf{F}$. However, there is no physical reason for that, and we proposed in this paper to consider the other cases in which $-\mathbf{w}_{\alpha}$ would be the shear-image of a reticular direction that is not necessarily $\mathbf{w}_{\gamma}$, i.e., in which $-\mathbf{w}_{\alpha}=\mathbf{t}_{\gamma}^{\prime}$ where $\mathbf{t}_{\gamma}$ is a reticular direction of the same $\mathbf{p}$-layer, i.e., $\mathbf{p}^{\mathbf{t}} \cdot \mathbf{t}=\mathbf{p}^{\mathbf{t}} \cdot \mathbf{w}=q$. In this mode, the shear direction becomes $\mathbf{d}=\mathbf{t}^{\prime}-\mathbf{t}$. With Friedel's hypothesis $\mathbf{t}=\mathbf{w}$, and since $\mathbf{w}^{\prime}+\mathbf{w}$ is parallel to $\mathbf{n}$, this mode was called "normal". In the case where $\mathbf{t} \neq \mathbf{w}$, and $\mathbf{t}^{\prime}+\mathbf{t}$ is not parallel anymore to $\mathbf{n}$, the mode was called "tilted". The tilted mode allowed us to explain with the index $q=1$ some twins without shuffle previously and oddly reported with a twin index $q=2$. The details of the calculations of the transformation matrices for the normal mode are given in Sections 2 and 3, and their generalization to the tilted modes are in 
Section 4. An important step that allowed us to establish these matrices quite automatically was the introduction of the supercell matrices and the general Equation (18).

The second novelty of the paper is the way to consider the type II twins. In the normal mode, the distortion matrix of a type I twin is given by Equation (19) $\mathbf{F}^{\gamma}=\mathbf{I}+\frac{1}{\mathbf{p}^{\mathbf{t}} \cdot \mathbf{w}}\left(\mathbf{d} \cdot \mathbf{p}^{\mathbf{t}}\right)$ where the twinning plane $\mathbf{p}$ is rational, and $\mathbf{w}$ is a rational direction of the $q$ th layer of the plane $\mathbf{p}$ that will also become a $\mathbf{w}$ direction of the twin. Generally, in literature, the plane $\mathbf{p}$ is noted $\mathbf{K}_{1}$, the vector $\mathbf{d}$ is parallel to $\boldsymbol{\eta}_{1}$, and the direction $\mathbf{w}$ is $\eta_{2}$. The plane usually noted $\mathbf{K}_{2}$ is the plane that contains $\mathbf{w}$ and that is only rotated by the shear (it would be fully invariant if the rotation were compensated). To our knowledge, literature always introduce type II twins as the conjugate twins of type I twins. Once a type I twin is defined from $\mathbf{p}=\mathbf{K}_{1}$ and $\mathbf{w}=\eta_{2}$, its other twinning elements $\mathbf{K}_{2}, \mathbf{d} / / \boldsymbol{\eta}_{1}$ are calculated, and the type II is deduced with $\mathbf{p}=\mathbf{K}_{2}$ and $\mathbf{d} / / \eta_{2}$. In the dyadic product $\mathbf{d} \cdot \mathbf{p}^{\mathbf{t}}$ of type I twin, the vector $\mathbf{p}$ is rational, but $\mathbf{d}$ is not necessarily rational, and in that of type II twins, the vector $\mathbf{d}$ is rational but $\mathbf{p}$ is not necessarily rational. Introducing type II twins as conjugates of type I twins hides an important concept. We showed that type II twins can be imagined exactly as type I twins by considering the reciprocal lattice instead of the direct lattice. Type II twins are a simple shear on a rational plane of the reciprocal space, and they can be treated with the same formalism as type I twins, independently of them. The direction $\mathbf{w}$ in the $q$-layer of the $\mathbf{p}$-plane used to build the supercell for the type I twins is replaced for the type II by the $g$ direction in the $q^{*}$-layer of the $\mathbf{d}$-plane, where $g$ is a rational direction of the reciprocal lattice (i.e., a reticular plane of the direct lattice) and $\mathbf{d}$ is a rational plane (i.e., a reticular direction of the direct lattice). The distortion matrix of type II twins is calculated exactly as for type I twins; they are expressed in the reciprocal space by $\mathbf{F}^{\gamma^{*}}=\mathbf{I}+\frac{1}{\mathbf{d}^{t} \cdot g}\left(\mathbf{p} \cdot \mathbf{d}^{\mathbf{t}}\right)$. Once determined, the type II shear can be expressed in the direct space by considering that $\mathbf{F}^{\gamma}=\left(\mathbf{F}^{\gamma^{*}}\right)^{-\mathbf{t}}$. Besides these two new complements to the classical Friedel's theory of twinning, we would like to take the opportunity of this study to discuss other points.

\subsection{Are the "Transformation Twins" Really Twins?}

In the paper we have only considered the deformation twins and the growth twins. In literature, there is a third category called "transformation twins". This last term is often taken as synonymous of "variants" or "domains" despite the fact that a simple observation generally permits to distinguish variants from twins. The variants inherited from a phase transformation appear as intricate laths or plates forming a complex microstructure, whereas the growth twins are isolated crystals in contact with a parent crystal, and the deformation twins appear as isolated plates or lenticles inside a parent host. The use of the term "twin" in place of "variants" dates from Friedel, and it was popularized by the Phenomenological Theory of Martensite Crystallography (PTMC). This theory, in Wechsler, Liebermann and Read's version [32] and its further developments [33-35], assumes that a twin relation exists between the variants. This hypothesis is completely admitted in the community of shape memory alloys. However, we have a very strong reservation on the PTMC and this basic hypothesis, as we detailed in [36], and we reject the idea that the variants are necessarily linked by a twin relation. Despite what is sometimes claimed, this assumption has never been proved experimentally, and it is not in agreement with some algebraic considerations. Let us explain them.

First, we consider the $r \rightarrow \alpha$ phase transformation, where the parent crystal $\checkmark$ and the daughter crystal $\alpha_{1}$ are linked by specific misorientation T. Because of the symmetries of the parent crystal $\gamma$, there are different daughter crystals equivalent to $\alpha_{1}$ that are called "variants". We call $\mathbb{G}^{\gamma}$ and $\mathbb{G}^{\alpha}$ the point group of the parent and daughter phases, respectively. The variants are given by the cosets $\alpha_{i}=g_{i}^{\gamma} \mathbb{H}_{\mathrm{T}}^{\gamma}$ where $g_{i}^{\gamma}$ are symmetry matrices of $\mathbb{G}^{\gamma}$, and $\mathbb{H}_{\mathrm{T}}^{\gamma}$ is the subgroup of the symmetries that are common to the parent crystal and the daughter crystal $\alpha_{1}$ :

$$
\mathbb{H}_{\mathrm{T}}^{\gamma}=\mathbb{G}^{\gamma} \cap \mathbf{T} \mathbb{G}^{\alpha} \mathbf{T}^{-1}
$$


Each variant $\alpha_{i}$ is misorientated with the parent crystal by a set of equivalent matrices $\alpha_{i} T$. The number of different variants $\alpha_{i}$ is given by Lagrange's formula [23,37]:

$$
N_{\mathrm{T}}^{\alpha}=\frac{\left|\mathbb{G}^{\gamma}\right|}{\left|\mathbb{H}_{\mathrm{T}}^{\gamma}\right|}
$$

The misorientations between the variants $\alpha_{i}$ and $\alpha_{j}$ are given by the sets of matrices $\mathbf{T}^{-1} \alpha_{i j} \mathbf{T}$, made with the double-cosets $\alpha_{i j}=\left(\alpha_{i}\right)^{-1} \alpha_{j}=\mathbb{H}_{\mathrm{T}}^{\gamma} g_{i j}^{\gamma} \mathbb{H}_{\mathrm{T}}^{\gamma}$ with $g_{i j}^{\gamma}=\left(g_{i}^{\gamma}\right)^{-1} g_{j}^{\gamma}$. The number of distinct misorientations between the variants is given by Burnside's formula (see [37]).

Deformation and growth twinning also imply a specific misorientation $\mathbf{T}$ between the parent crystal $\Upsilon$ and its daughter twin crystal $\alpha_{1}$. Both $\Upsilon$ and $\alpha$ crystals are constituted of the same phase. Because of the symmetries of the parent crystal, there are different equivalent daughter twins. As for phase transformations, the twin variants are given by the cosets $\alpha_{i}=g_{i}^{\gamma} \mathbb{H}_{\mathrm{T}}^{\gamma}$ where $\mathbb{H}_{\mathrm{T}}^{\gamma}$ is given by Equation (33) with $\mathbb{G}^{\gamma}=\mathbb{G}^{\alpha}$. Each twin variant $\alpha_{i}$ is misorientated with the parent crystal by a set of equivalent matrices $\alpha_{i} \mathbf{T}$. The number of twin variants $\alpha_{i}$ is also given by Lagrange's formula (34).

The difference between the deformation/growth twins and the transformation variants is now clearer. The misorientations between a parent and its equivalent twins are given by the simple cosets $\alpha_{i} \mathbf{T}$, and the number of twins is given Lagrange's formula, whereas the misorientations between the variants are given by the double cosets $\mathbf{T}^{-1}\left(\alpha_{i}\right)^{-1} \alpha_{j} \mathbf{T}$ and their number is given Burnside's formula. In twinning, the parent and its twin are in direct contact, and this direct relation is expressed by a simple coset. In the case of a phase transformation, the variants grow from the parent phase independently of each other; when they meet each other the relation between them is indirect and expressed by double-cosets. PTMC made the hypothesis that one variant can be directly transformed by twinning into another one by stress: $\alpha_{i} \rightarrow \alpha_{j}$; this phenomenon was called "variant reorientation" or "detwinning", but we think that actually the transformation is indirect and mediated by the parent phase: $\alpha_{i} \rightarrow \gamma \rightarrow \alpha_{j}$. Let us come back to the origin of our difference of point of view. In the case of deformation/growth twinning, the matrix $\mathbf{T}$ is necessarily a mirror symmetry or a $180^{\circ}$ rotation, whereas for transformations variants the matrices forming the double cosets $\mathbf{T}^{-1}\left(\alpha_{i}\right)^{-1} \alpha_{j} \mathbf{T}$ do not necessarily contain these 2 -fold operations. The 2 -fold operators are called "ambivalent", and the others are called "polar" [37-39]). PTMC assumes that all the operators are of 2-fold type; it ignores the existence of the polar operators. How is it possible? This is because in its latest versions PTMC implicitly assumes that there is not a unique misorientation T. PTMC considers $\mathbf{T}$ as secondary minor adjusting parameter deduced from the compatibility criterion between the "correspondence" variants (which are in fact "stretch" variants [23]). Here again, we disagree. Experiments show that there is a unique orientation $\mathbf{T}$, with possibly some continuous slight misorientations around $\mathbf{T}$ due to the plastic accommodation of the distortion [40-45]. We consider that the compatibility obtained by variant coupling is not essential because dislocations can act as additional accommodation means. We believe that a unique orientation $\mathbf{T}$ should be the core of the crystallographic models of phase transformation. The great metallurgist Nishiyama was convinced in the 1950s to give up his earlier model of martensitic transformations in steels [46] because of the TEM observations he made of some twins in steels [47], and later he became an ardent partisan of the PTMC in his book [48]. However, he timidly conceded at the end of his carrier in a one-page comment [49]: "it is adventurous to deduce any conclusion associated with more fundamental laws from the invariant plane hypothesis. It is desirable to induce the fundamental law from the data of some phenomena with least deviation, such as the orientation relationships". It is unfortunate that he could not continue gown this path, and that his comment disappeared into oblivion.

To conclude this section, we strongly recommend keeping the term "twin" for real growth or deformation twins, and use the clearer terms "variants" or "domains" for the daughter products created by a phase transformation. 


\subsection{Can 3, 4 and 6-Fold Operations be Twin Operations?}

Friedel always considered that twinning operations are not limited to the 2-fold operations (mirror symmetry or $180^{\circ}$ rotation for the twin edifice); he argued that 3, 4 and 6-fold operations should also be included in the theory. Despite our great admiration for his work, we will not follow him on this topic, and we prefer to adopt Mügge's point of view that there is no need of these higher order operations. Friedel showed with many examples in mineralogy that twinning is a matter of metrics and lattices, not of symmetry. Thus, it seems contradictory to introduce ex nihilo some crystal symmetries in the twining theory. They look very similar to the pseudo-symmetries derived from Mallard's hypothetical cubic phase that Friedel still discarded. It seems that, more than one century after Mügge and Friedel, the debate about the high-order operations is not yet closed (see [1]). We think very possible that the cases of complex edifices that look like high-order twins are not twins but variants inherited from a real high temperature phase during cooling (see previous Section). The observed 3, 4 or 6-fold symmetry would be that of the parent phase, and the variants would come in contact during their growth from the parent crystal, without direct transformation (by shear or misfit reduction) between the lattices of the variants. The other possibility is that they are not the result of a simple shear and would be special types of epitaxial twins (see next Section).

\subsection{Beyond Dogmas}

Science is very conservative and slowly advances. This makes it strong and reliable. However, it should always be remembered that science works with models that are based on hypotheses and paradigms, not on dogmas. We should remain open to new ideas as far as they are in agreement with the observations, and we should give up the old models, or at least make them evolve, if they cannot explain some experimental facts. Models and theory should never substitute the experience. Let us propose some original paths to explore for future research.

\subsubsection{Epitaxial Twinning}

Clearly, the crystallographic theory of twinning does not explain all the observations, even with the complements brought in this paper. Friedel was not completely satisfied; his model based on the lattice or multiple lattice could describe many growth twins of minerals, but some of them remained

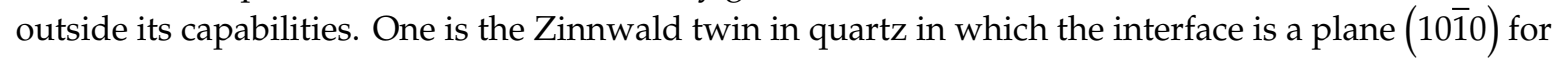
the parent and $(10 \overline{1} 1)$ for its twin. The fact that the interface cannot be indexed similarly in the parent and its twin is difficult to understand within the classical Friedel's theory. It seems that they are not type II twins, even if we have not yet checked this point. Quartz also produces unconventional twins such as the Lötshental twins, the Zyndel twins and the Seedorf twins [1]. To explain them, Friedel [50] at the end of his life proposed a model based on diperiodic multiple "lattice" that replaces the triperiodic one, but these developments were largely ignored by the scientific community, probably because of the rareness of these unconventional twins. For the last decade, we have used the atomic hard-sphere assumption to establish some simple crystallographic models of martensitic transformations in steels and other alloys [40-45] and of deformation twinning in magnesium alloys [23,30,51,52]. We came to conclude that the habit plane is not fully invariant during the lattice distortion. For example, in

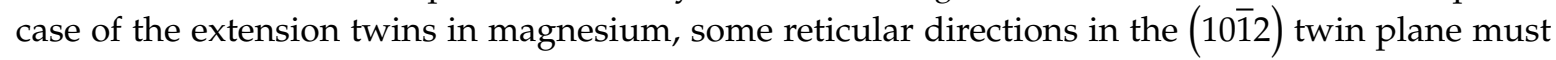
elongate to leave some $\mathrm{Mg}$ atoms go through the plane, before coming back to their initial length once the distortion is complete [30]. Thus, the lattice distortion is not a simple shear mechanism in it is continuity; it is a simple shear only if the pictures of the initial and final states are considered, and if the movie of the atomic displacements during the process if ignored. After this theoretical work, the experimental discovery an unconventional deformation twin in magnesium in which the interface is $(21 \overline{3} 2)$ in the host parent and $(01 \overline{1} 2)$ in the twin [52] came without surprise (but not without satisfaction). At that time, we were not yet aware that Friedel already encountered unconventional growth twins in mineralogy. If twinning is not a simple shear mechanism, what could it be? A generalized theory of 
twinning should be in agreement with the classical twinning cases, but also with the unconventional ones in which the interface plane is not invariant. The general character of twinning comes from the fact that the accommodation is spread in whole volume. For deformation twins, the energy required to create this accommodation volume is brought by the work of the external stress $W=\sigma_{i j} \varepsilon_{i j}$. As the invariance condition of the interface plane is not required anymore, new forms of distortion matrices $\mathbf{F}$ are possible. A necessary condition is that the volume is conserved, i.e $\operatorname{Det}(\mathbf{F})=1$. Additional conditions must substitute the previous "invariant plane" one; and among them, one could be that $\mathbf{F}$ minimizes the misfits in volume with the host parent by adopting an epitaxy orientation relationship. For steels we proposed that the habit plane should be an unrotated plane [43], i.e., it should be an eigenvector of $\mathbf{F}^{*}$; and this condition could probably be acceptable to define a part of the epitaxial relation. A wide range of new distortion matrices is now open. An example of such possible epitaxial distortion is shown in Figure 15a. The general theory with its mathematics remains to be built, and more experimental results on the unconventional twins should be collected.
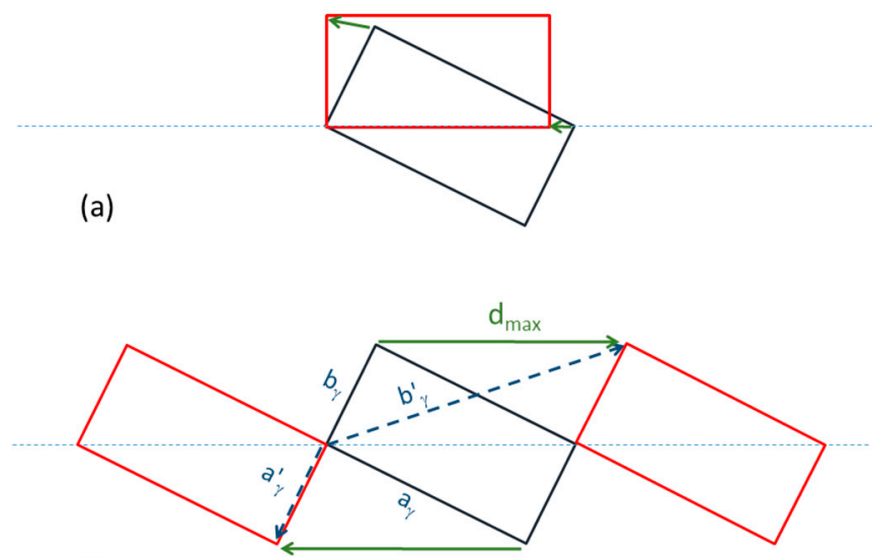

(b)

Figure 15. Unconventional twins. (a) Epitaxial twin. The distortion matrix $\mathbf{F}$ is not a simple shear. (b) Iso-orientation twin. The distortion matrix $\mathbf{F}$ is a shear but the orientation $\mathbf{T}$ is the identity.

\subsubsection{Iso-orientation Twinning}

Even if the simple shear hypothesis is maintained, there is another assumption in the usual crystallography of deformation twinning that is implicit or admitted as "obvious", but that could be discussed: it is said that the twinning plane $\mathbf{p}$ should not be a mirror plane of the crystal lattice, and its normal $\mathbf{n}$ should not be a 2-fold symmetry of the individual crystal lattice. Such cases however exist for growth twinning; they correspond to "twinning by merohedry", in which $\mathbf{T}$ is a mirror symmetry of the lattice but not of the crystal. For deformation twinning, a distortion that would lead to the same lattice orientation was excluded. We think that this restrictive hypothesis results from a confusion between the orientation and the distortion. Actually, iso-orientation twinning can be imagined for $\mathbf{T} \in \mathbb{G}^{\gamma}$ with the only condition that the shear vector $\mathbf{d}$ is a reticular direction. The cases would be equivalent to $\mathbf{T}=\mathbf{I}$ (identity matrix) and $\mathbf{F}=\mathbf{C}$. An example is given in Figure $15 \mathrm{~b}$. The twin would be "invisible" by X-ray diffraction or by Electron BackScatter Diffraction (EBSD) mapping, or at least would be detectable only the traces left by the accommodation dislocations and by the continuous rotation fields they generate. These rotation fields take the form of continuous features in the pole figures, as already observed for martensite in steels [40] and for the extension twins in magnesium [30]. The possibility of iso-orientation twinning would be equivalent to a glide of perfect dislocations in regularly stacked in layers parallel to the twinning plane. As already proposed by Friedel [20], it is possible that the unclear phenomena called "kinking" ("knickung" in German, as introduced by Mügge, and later translated by "pliage en genou" in French) is the manifestation of such iso-T shears. Kinking is a macroscopic and fast bending deformation of the crystal without the creation of new distinct 
orientations. A schematic representation of the lattice change during kinking is shown in Figure 16. Nowadays, kinking and shear bands are considered to result from a collective and brutal glide glides of dislocations (Figure 16a), but we prefer following Friedel to conceive kinking as a kind of iso-T twinning (Figure 16b). Friedel clearly proposed that "glide is nothing else than a mechanical twin by which the crystal is transformed into itself" [20]. This point of view has completely been forgotten because of the great success of dislocations from 1940s till now.

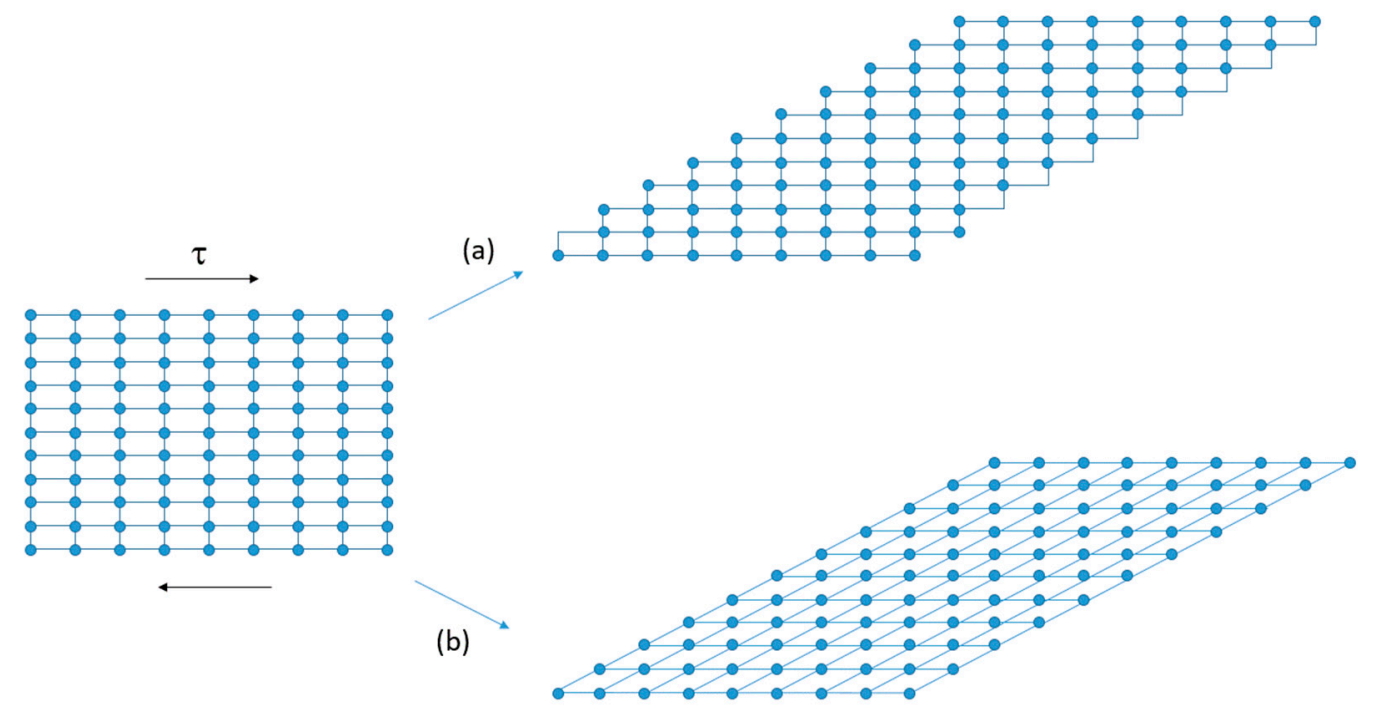

Figure 16. Two interpretation of the kinking phenomenon ("pliage en genou" in French). (a) By a sequential and well-ordered movement of perfect dislocations. The origin of the dislocations is difficult to explain. (b) By an iso-orientation shear. The nodes of the lattices (blue disks) are in the same positions in $(\mathbf{a}, \mathbf{b})$.

We do not contest that dislocations exist; they react together; they explain many physical and mechanical properties; but we do not believe that they explain everything in materials science. To our point of view, dislocations are not relevant to explain collective effects such as martensitic transformations and deformation twinning. They are the consequence (the defects) created by the transformations, but they are not their cause and they should not be taken as the starting point of the models. As we already argued in [36], all the models of martensitic transformation and deformation twinning that are based on sequential and periodic glides of partial "interface dislocations" remain mute on the origin of these dislocations. A complex "pole mechanism" was imagined but never confirmed experimentally.

Explaining the macro/mesoscopic phenomenon of kinking and deformation twinning by a lattice distortion driven by the interaction work $\mathrm{W}=\boldsymbol{\sigma}_{i j} \varepsilon_{i j}$ is more natural to us than imagining individual and correlated glides under the Peach-Koehler force of dislocations created by an unknown or highly speculative process. The ideao iso-orientation lattice shear has been discarded to the profit of dislocation-based plasticity because it is usually believed that it is energetically impossible for the atoms to move collectively by a macroscopic shear. This is the famous Frenkel's argument based on an incorrect demonstration and against which we proposed a wave-like propagation of phase transformation [36]. In metallurgy and in crystallography, the belief that iso-T deformation can only be obtained by dislocation glide is so strong that mathematics of crystallography use the semi-direct Seitz notation $\{\mathbf{F} \mid \mathbf{t}\}$ of affine operations in order to separate the linear application $\mathbf{F}$ from the translation t. However, some researchers have introduced the "global group" that contains all the operations that preserve the lattice with its orientation $[34,35]$ and that contains the iso-T shear distortion we are describing. We remained very cautious on the real existence of the "global" group (see Section A2 of [23]) because the iso-T shear would indeed imply high shear values and important accommodation 
energies, but the wave-like process we proposed in [36] could allow in some rare cases such large shears to be favored by the work of the external stress.

Let us take the risk to go even further by generalizing the idea of iso-orientation shear to usual gliding. If only the initial and final states are considered, imagining dislocations as incompatibility defects resulting from an iso-orientation shear is very similar the usual view of dislocation gliding, as illustrated in Figure 17.

Imagining gliding as localized nano-shear band could have some advantages over the dislocation model to explain some mesoscopic phenomena. The glide in some bcc metals and some minerals is easy in one direction and impossible in the reverse direction. This fact and other non-Schmid behaviors are difficult to explain with dislocations, even by considering their complex core structure. They are simpler to understand by seeing them as cases of iso-T twinning with $q>1$ for which the shuffles of the atoms inside the supercell depend on the direction of the applied shear.
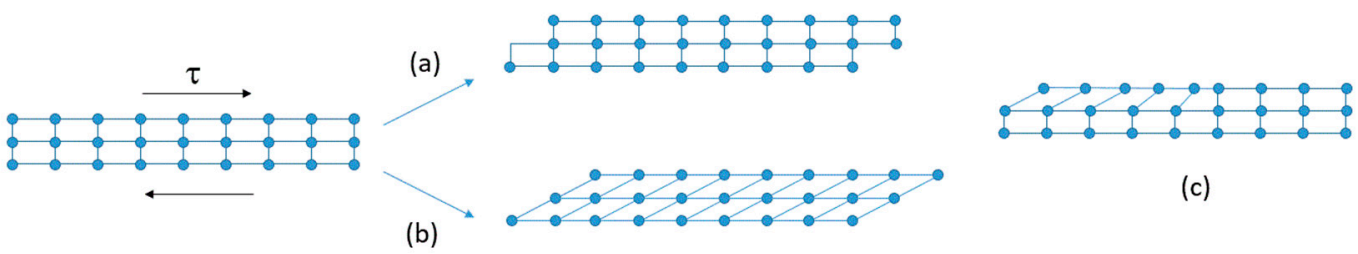

(c)

(b)
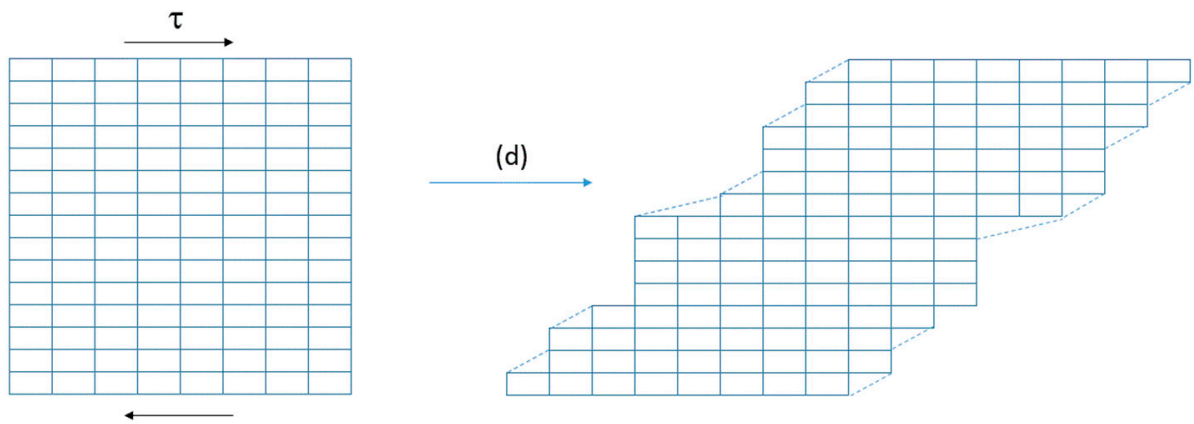

Figure 17. Similarities between glide and twinning. (a) Usual view on glide, the upper lattice is translated relatively to the lower part. (b) Same final state obtained by iso-orientation twinning. (c) Dislocations are the frontiers between sheared and a non-yet sheared parts of the crystal. (d) Plasticity is usually considered as a consequence of glides, but it could be equivalently explained localized sheared bands.

\section{Conclusions}

Deformation and growth twins can be described with the same mathematics. The concept of lattice misfit and obliquity for the growth twins is indeed quite equivalent to that of shear incompatibility for the deformation twins. The paper recalls the hypotheses on which the theory has been built over the last 130 years with Mügge, Mallard, and Friedel works, and later with Bilby, Bevis and Crocker's developments (Section 1). The three transformation matrices (distortion $\mathbf{F}$, orientation $\mathbf{T}$, correspondence $\mathbf{C}$ ) are determined in dyadic forms, first by synthesizing the various geometrical/algebraic results of literature (Section 2), and secondly, by proposing a systematic method that uses the supercell matrices (Section 3). Then, two important complements to the usual theory were proposed.

(1) The usual theory only considers the cases in which $\mathbf{F}$ and $\mathbf{T}$ play a similar role (on the reticular direction w). This assumption is justified for twinning with low obliquity/shear values, but is not relevant for larger values. We showed in Section 4 that in such cases $\mathbf{F}$ and $\mathbf{T}$ should be uncorrelated, with $\mathbf{T}$ playing on $\mathbf{w}$, and $\mathbf{F}$ acting on another reticular direction $\mathbf{t}$ pointing to a node of the same layer as that of $\mathbf{w}$. The usual twinning mode was called "normal" and the other modes were called "tilted". The normal and tilted modes constitute "complementary" twins. The 
tilted modes explain with an index $q=1$ some twins without lattice shuffling previously reported with $q=2$ in literature.

(2) Type II twins have always been introduced as the conjugates of type I twins, which does not permit to capture their true nature. We showed in Section 5 that type II twinning is a simple shear on a reticular plane of the reciprocal lattice, exactly as type I twining is a simple shear on a reticular plane of the direct lattice. Type II twins can be determined independently of the type I twins, with the same mathematics.

In the Section 6, we explained why the variants inherited from a phase transformation should not be called "transformation twins". We also showed that, even with the complements proposed in the paper, the crystallography of twinning needs more generalization. As Friedel, we think that twinning is fundamentally not a simple shear mechanism. The non-shear nature of twinning is particularly clear in the cases of the Zinnwald growth twin in which the interface is $(10 \overline{1} 0)_{\text {parent }} / /(10 \overline{1} 1)_{\text {twin }}$, and for the unconventional deformation twin we recently observed in magnesium for which the interface is $(21 \overline{3} 2)_{\text {parent }} / /(01 \overline{1} 2)_{\text {twin }}$. These "epitaxial twins" are not yet mathematically formalized. We also follow Friedel to consider that kinking could be a type of "iso-orientation twinning".

When, more a century ago, Mügge, Mallard and Friedel proposed models of twinning based only the lattice and its metrics, the existence of the atoms was still discussed and the structure of the motif was very vague. Their approaches were very successful, but not yet complete, and Friedel knew it. It is now great time to rehabilitate the atoms and propose general forms of lattice distortions that are compatible with their sizes, or more precisely with their interatomic potentials and the energetic gaps of the intermediate states. Crystallography needs physics and mechanics to complete the twinning models. It is also important to re-analyze without pre-conceived opinion the numerous cases of twinning and kinking encountered in metallurgy and mineralogy with modern experimental tools, such as in-situ X-ray or neutron diffraction, and EBSD.

Funding: This research received no external funding.

Acknowledgments: I would like to show all my gratitude to Prof. Roland Logé, director of LMTM, for his trust and the freedom he gives me to work on this type of "old school" research, and to EPFL and PX group for the laboratory funding. The anonymous reviewers are also thanked for their judicious advice and suggestions.

Conflicts of Interest: The authors declare no conflict of interest.

\section{Appendix A}

We consider the 2D rectangular crystal with its right-hand crystallographic basis $\left(\mathbf{a}_{\gamma}, \mathbf{b}_{\gamma}\right)$ and its twin with its left-hand crystallographic basis $\left(\mathbf{a}_{\alpha}, \mathbf{b}_{\alpha}\right)$. The normal and tilted twinning modes leading to the twinned crystal are represented in Figure A1(a) and Figure A1(b), respectively. What are the transformation matrices and the shear amplitudes for these two modes? In order to simplify the notation, we assume that $\mathrm{a}_{\alpha}=1$ and that $\mathrm{b}_{\alpha} / \mathrm{a}_{\alpha}=r$ with $r \leq 1$.

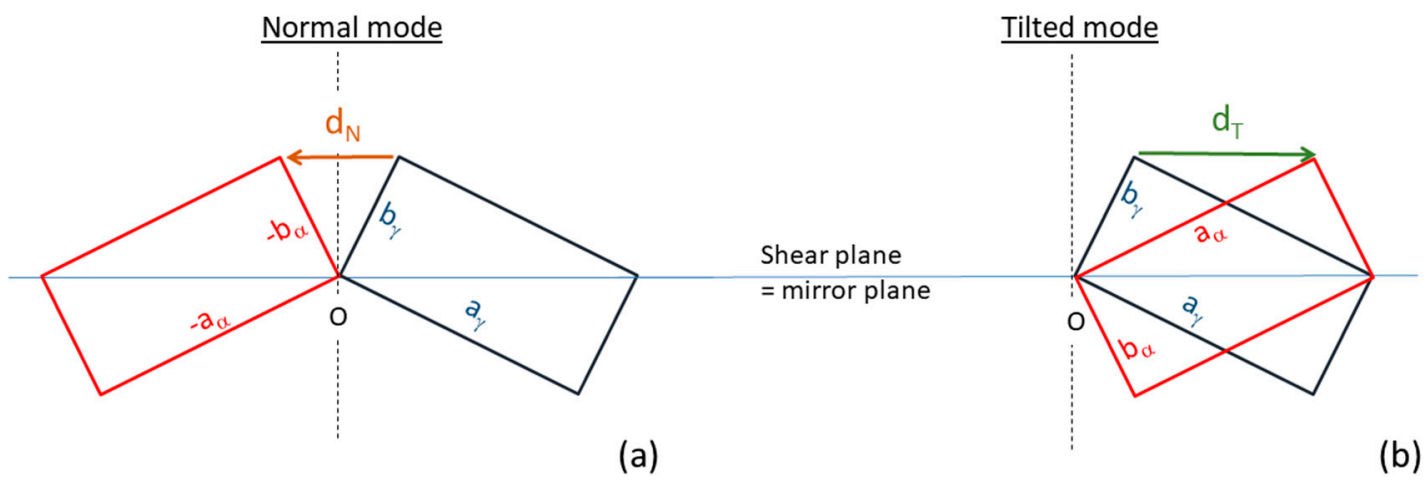

Figure A1. Twinning of a rectangle along its diagonal with $q=1$. (a) Normal mode. (b) Tilted mode. 
The twinning plane is $\mathbf{p}=\left(\begin{array}{c}-1 \\ 1\end{array}\right)$ and the twinning direction $\mathbf{d}$ is parallel to $\mathbf{d}_{\max }=\left[\begin{array}{l}1 \\ 1\end{array}\right]$. Please note that the amplitude of $\mathbf{d}$ is not yet know. Let us consider Figure A2. It gives the relations: (1) $d_{\text {max }}=\sqrt{1+r^{2}},(2) h \cdot d_{\max }=r$ (surface of the rectangle), (3) $h^{2}+e^{2}=r^{2}$. From them, it can be shown that the shear value for the normal mode is:

$$
s_{\mathrm{N}}=\frac{d_{N}}{h}=\frac{2 e}{h}=2 r
$$

and the shear value for the tilted mode is:

$$
s_{\mathrm{T}}=\frac{d_{T}}{h}=\frac{1}{r}-r
$$

The shear values are plotted as functions of $r$ in Figure A3.

Note that $s_{\mathrm{N}}+s_{\mathrm{T}}=s_{\max }$ with $s_{\max }=\frac{d_{\max }}{h}$. The shear amplitude of the two modes are equal for a rectangle with the metric ratio $r_{c}=\frac{1}{\sqrt{3}}$. If one considers that the lowest shear is favored, the twinning mode will be normal for $r \leq r_{c}$ and tilted for $r \geq r_{c}$.

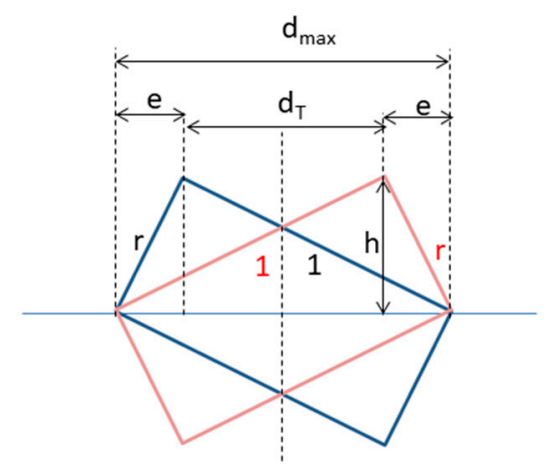

Figure A2. Geometrical values related to Figure A1.

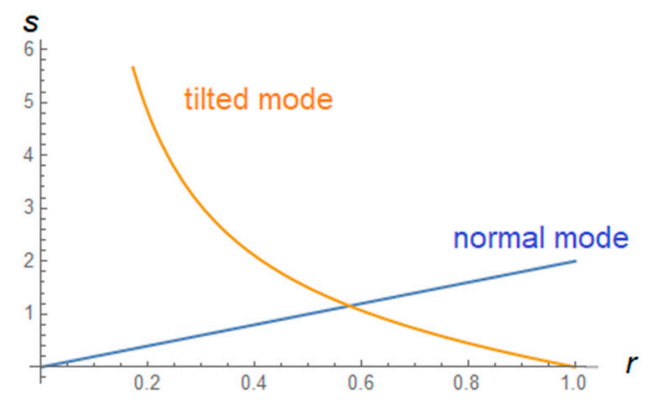

Figure A3. Shear amplitude of the normal and tilted mode as functions of the metric $r$.

The metric tensor of the rectangle is $\mathcal{M}=\left(\begin{array}{ll}1 & 0 \\ 0 & r^{2}\end{array}\right)$ and its reciprocal $\mathcal{M}^{*}=\left(\begin{array}{cc}1 & 0 \\ 0 & 1 / r^{2}\end{array}\right)$. They allow to find the norm of the vectors $\left\|\mathbf{d}_{\text {max }}\right\|=\sqrt{\mathbf{d}_{\text {max }}^{\mathrm{t}} \boldsymbol{M} \mathbf{d}_{\text {max }}}=\sqrt{1+r^{2}}$, and $\|\mathbf{p}\|^{*}=\sqrt{\mathbf{p}^{\mathrm{t}} \mathcal{M}^{*} \mathbf{p}}=\frac{\sqrt{1+r^{2}}}{r}$. The normalized vectors are $\tilde{\mathbf{d}}=\frac{\mathbf{d}_{\max }}{\left\|\mathbf{d}_{\max }\right\|}= \pm \frac{1}{\sqrt{1+r^{2}}}\left[\begin{array}{l}1 \\ 1\end{array}\right]$ and $\tilde{\mathbf{p}}=\frac{\mathbf{p}}{\|\mathbf{p}\|^{*}}=\frac{r}{\sqrt{1+r^{2}}}\left(\begin{array}{c}-1 \\ 1\end{array}\right)$.

The shear matrix for each mode is now deduced from Equation (5) $\mathbf{F}=\mathbf{I}+s(\tilde{\mathbf{d}} \cdot \tilde{\mathbf{p}})$ with $s=s_{\mathrm{N}}$ and $\tilde{\mathbf{d}}=-\frac{1}{\sqrt{1+r^{2}}}\left[\begin{array}{l}1 \\ 1\end{array}\right]$ for the normal mode, and $s=s_{\mathrm{T}}$ and $\tilde{\mathbf{d}}=\frac{1}{\sqrt{1+r^{2}}}\left[\begin{array}{l}1 \\ 1\end{array}\right]$ for the tilted mode. 
They are $\mathbf{F}_{\mathrm{N}}=\frac{1}{1+r^{2}}\left[\begin{array}{cc}1+3 r^{2} & -2 r^{2} \\ 2 r^{2} & 1-r^{2}\end{array}\right]$ and $\mathbf{F}_{\mathrm{T}}=\frac{1}{1+r^{2}}\left[\begin{array}{cc}2 r^{2} & 1-r^{2} \\ r^{2}-1 & 2\end{array}\right]$. It can be checked that both matrices leave the direction $\left[\begin{array}{l}1 \\ 1\end{array}\right]$ invariant.

Another and more automatic way to determine the shear matrices $\mathbf{F}_{\mathrm{N}}$ and $\mathbf{F}_{\mathrm{T}}$ consists in using Equation (19) instead of Equation (5). For normal mode $\mathbf{F}_{\mathrm{N}}$ is calculated from $\mathbf{d}$ given by Equation (6) and $\mathbf{w}=\mathbf{b}_{\gamma}$, and for the tilted mode $\mathbf{F}_{\mathrm{T}}$ is calculated by Equation (21) with $\mathbf{w}=\left[\begin{array}{l}0 \\ 1\end{array}\right]$ and $\mathbf{t}=\left[\begin{array}{l}-1 \\ 0\end{array}\right]$. Following this method, there is no need to calculate the shear amplitudes. The values $s_{\mathrm{N}}$ and $s_{\mathrm{T}}$ can be determined afterwards, by using Equation (12).

The correspondence matrices are determined by considering Figure A4. In the normal mode, the shear transforms the basis vectors of the parent crystal $\mathbf{a}_{\gamma} \rightarrow \mathbf{a}_{\gamma}^{\prime}=\mathbf{a}_{\alpha}+2 \mathbf{b}_{\alpha}$ and $\mathbf{b}_{\gamma} \rightarrow \mathbf{b}_{\gamma}^{\prime}=-\mathbf{b}_{\alpha}$; the correspondence matrix is thus $\mathbf{C}_{N}=\left[\begin{array}{cc}1 & 0 \\ 2 & -1\end{array}\right]$. In the tilted mode, the shear transforms the basis vectors of the parent crystal $\mathbf{a}_{\gamma} \rightarrow \mathbf{a}_{\gamma}^{\prime}=\mathbf{b}_{\alpha}$ and $\mathbf{b}_{\gamma} \rightarrow \mathbf{b}_{\gamma}^{\prime}=\mathbf{a}_{\alpha}$; the correspondence matrix is thus $\mathrm{C}_{\mathrm{N}}=\left[\begin{array}{ll}0 & 1 \\ 1 & 0\end{array}\right]$. Their determinant is -1 because of the convention that twinning is a mirror symmetry of lattices. The correspondence matrices and the metric tensors could be used to find again the shear amplitudes thanks to Bevis and Crocker's Equation (11), and the calculations confirm the values of $s_{N}$ and $s_{\mathrm{T}}$ previously determined.

The orientation matrix is the same in both modes. It is given by Equation (8). Calculations show that $\mathbf{T}=\frac{1}{1+r^{2}}\left[\begin{array}{cc}1-r^{2} & 2 r^{2} \\ 2 & 1-r^{2}\end{array}\right]$. One can check the master equation $\mathbf{C}=\mathbf{T} \cdot \mathbf{F}$.

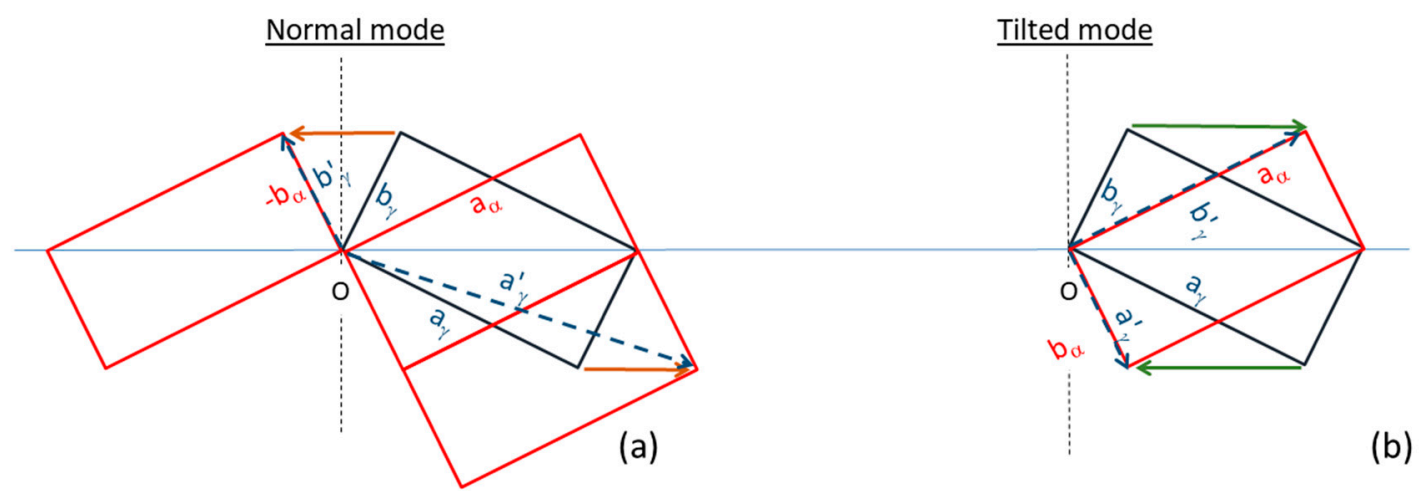

Figure A4. Scheme to determine the correspondence matrices in (a) a normal mode, and (b) a tilted mode.

\section{References}

1. Boulliard, J.-C. Le Cristal et ses Doubles; CNRS Editions: Paris, France, 2010.

2. Haüy, R.J. Traité de Minéralogie 1801. Five Volumes. Available online: http://catalogue.bnf.fr/ark:/12148/ cb373669857.public (accessed on 6 February 2020).

3. Thomson, W.; Tait, P.G. Treatise on Natural Philosophy; Cambridge University Press: Cambridge, UK, 1867; Volume I (1) 170-171, pp. 105-106, (pp. 123-124 in the second edition 1879, §§149-150 in the Elements of Natural Philosophy, a simplified version of the Treatise).

4. Mügge, O. Ueber homogene Deformationen (einfache Schiebungen) an den triklinen Doppelsalzen BaCdC14.4aq. Neues Jahrb. Für Mineral. Geol. Und Palaeontol. Beil. 1889, 6, 274-304.

5. Mügge, O. Über durch Druck entstandenes Zwillinge von Titanit nach den Kanten [100] uund [10]. Jahrb. Für Mineral. Geol. Und Palaeontol. Beil. 1889, 2, 98-115, (+Taff.II). 
6. Hardouin Duparc, O.B.M. A review of some elements for the history of mechanical twinning centred on its German origins until Otto Mügge's K1 and K2 invariant plane notation. J. Mater. Sci. 2017, 52, 4182-4196. [CrossRef]

7. Cahn, R.W. Twinned Crystals. Adv. Phys. 1954, 3, 363-445. [CrossRef]

8. Reed-Hill, R.E.; Hirth, J.P.; Rogers, H.C. (Eds.) Deformation Twinning; Gordon \& Breach: New York, NY, USA, 1964.

9. Hall, E.O. Twinning and Diffusionless Transformations in Metals; Butterworths Scientific Publications: London, UK, 1954.

10. Christian, J.W.; Mahajan, S. Deformation Twinning. Prog. Mater. Sci. 1995, 39, 1-157. [CrossRef]

11. Kihô, H. The Crystallographic Aspect of the Mechanical Twinning in Metals. J. Phys. Soc. Jpn. 1954, 9, 739-747. [CrossRef]

12. Jaswon, M.A.; Dove, D.B. The Crystallography of Deformation Twinning. Acta Cryst. 1960, 13, $232-240$. [CrossRef]

13. Bilby, B.A.; Crocker, A.G. The Theory of the Crystallography of Deformation Twinning. Proc. R. Soc. Lond. A 1965, 288, 240-255.

14. Bevis, M.; Crocker, A.G. Twinning Shears in Lattices. Proc. R. Soc. Lond. A 1968, 304, 123-134.

15. Crocker, A.G.; Bevis, M. The crystallography of deformation twinning in titanium. In The Science Technology and Application of Titanium; Jaffee, R., Promisel, N., Eds.; Pergamon Press: Oxford, UK, 1970; pp. 453-458.

16. Mallard, E. Cours de minéralogie. Recueil de Données Cristallographiques et Physiques Concernant les Principales Espèces Minérales; Ecole des Mines de Paris: Paris, France, 1893.

17. Hahn, T.; Janovec, V.; Klapper, H. Bicrystals, twins and domain structures-A comparison. Ferroelectrics 1999, 222, 11-21. [CrossRef]

18. Nespolo, M.; Ferraris, G. Overlooked problems in manifold twins: Twin misfit in zero-obliquity TLQS twinning and twin index calculation. Acta Cryst. 2007, A63, 278-286. [CrossRef] [PubMed]

19. Friedel, G. Etudes sur les Groupements Cristallins; Société de l'Imprimerie Théolier: Fourneaux, France, 1904.

20. Friedel, G. Leçons de Cristallographie; Berger-Levrault: Paris, France, 1926.

21. Bollmann, W. Crystal Defects and Crystalline Interfaces; Springer: New York, NY, USA, 1970.

22. Hardouin Duparc, O.B.M. A review of some elements in the history of grain boundaries centered on Georges Friedel, the coincident 'site' lattice and the twin index. J. Mater. Sci. 2011, 46, 4116-4134. [CrossRef]

23. Cayron, $\mathrm{C}$. The transformation matrices (distortion, orientation, correspondence), their continuous forms and their variants. Acta Cryst. 2019, A75, 411-437.

24. Bhadeshia, H.K.D.H. Worked Examples in the Geometry of Crystals, 2nd ed.; Brookfield: London, UK, 1987.

25. Cayron, C. Screening the simple shear twins. J. Appl. Cryst. in preparation.

26. Pabst, A. Transformation of indices in twin gliding. Bull. Geol. Soc. Am. 1955, 66, 897-912. [CrossRef]

27. Andrews, K.W.; Johnson, W. Formulae for the transformation of indices in twinned crystals. Br. J. Appl. Phys. 1955, 6, 92-96. [CrossRef]

28. Feng, C.R.; Michel, D.J.; Crowe, C.R. Transformation of Miller indices in twinned structures. Philos. Mag. Let. 1989, 60, 137-140. [CrossRef]

29. Klassen-Neklyudova. Mechanical Twinning of Crystals; Consultant Bureau: New York, NY, USA, 1964.

30. Cayron, C. Hard-sphere displacive model of extension twinning in magnesium. Mater. Des. 2017, 119, 361-375. [CrossRef]

31. Spence, J.; Zuo, J.M. Electron Microdiffraction; Plenum: New York, NY, USA, 1992.

32. Wechsler, M.S.; Liebermann, D.S.; Read, T.A. On the theory of the formation of martensite. Trans. Aime 1953, 197, 1503-1515.

33. Ball, J.M.; James, R.D. Fine phase mixtures as minimisers of energy. Arch. Nat. Mech. Anal. 1987, 100, 13-52. [CrossRef]

34. Bhattacharya, K. Microstructure of Martensite, Why it Forms and how It Gives Rise to the Shape Memory Effect; Oxford Series on Materials Modelling; Oxford University Press: Oxford, UK, 2003.

35. Pitteri, M.; Zanzotto, G. Continuum Models for Phase Transitions and Twinning in Crystals; Chapman \& Hall/CRC: London, UK, 2003.

36. Cayron, C. Shifting the shear paradigm in the crystallographic models of displacive transformations in metals and alloys. Crystals 2018, 8, 181. [CrossRef]

37. Cayron, C. Groupoid of orientational variants. Acta Cryst. 2006, A62, 21-40. [CrossRef] [PubMed] 
38. Janovec, V. Group analysis of domains and domain pairs. Czech. J. Phys. B 1972, 22, 975-994. [CrossRef]

39. Janovec, V.; Dvorakova, E. The coset and double coset decomposition of the 32 crystallographic point groups. Acta Cryst. A 1989, 45, 801-802. [CrossRef]

40. Cayron, C.; Barcelo, F.; de Carlan, Y. The mechanism of the fcc-bcc martensitic transformation revealed by pole figures. Acta Mater. 2010, 58, 1395-1402. [CrossRef]

41. Cayron, C. One-step model of the face-centred-cubic to body-centred-cubic martensitic transformation. Acta Cryst. 2013, 69, 498-509. [CrossRef]

42. Cayron, C. EBSD imaging of orientation relationships and variants groupings in different martensitic alloys and Widmanstätten iron meteorites. Mater. Charac. 2014, 94, 93-110. [CrossRef]

43. Cayron, C. Continuous atomic displacements and lattice distortion during fcc-bcc martensitic transformation. Acta Mater. 2015, 96, 189-202. [CrossRef]

44. Cayron, C. Angular distortive matrices of phase transitions in the fcc-bcc-hcp system. Acta Mater. 2016, 111, 417-441. [CrossRef]

45. Baur, A.P.; Cayron, C.; Logé, R.E. $\{225\} \gamma$ habit planes in martensitic steels: From the PTMC to a continuous model. Sci. Rep. 2017, 7, 40938. [CrossRef]

46. Nishiyama, Z. X-Ray Investigation of the Mechanism of the Transformation from Face-Centred Cubic Lattice to Body-Centred Cubic. Sci. Rep. Tohoku Univ. 1934, 23, 637-668.

47. Nishiyama, Z.; Shimizu, K. On the sub-bands in a martensite plate. J. Electron. Microsc. 1956, 4, 51.

48. Nishiyama, Z. Martensite Transformation; Materials Science Series; Fine, M.E., Meshii, M., Waymann, C.M., Eds.; Academic Press: New York, NY, USA, 1978.

49. Nishiyama, Z. A comment on the phenomenological theory of the crystal habit of martensite. J. Less Com. Met. 1972, 28, 95. [CrossRef]

50. Friedel, G. Sur un nouveau type de macles. Bull. De La Société Française De Minéralogie 1933, 56, $262-274$.

51. Cayron, $\mathrm{C}$. A one-step mechanism for new twinning modes in magnesium and titanium alloys modelled by the obliquity correction of a $\left(58^{\circ}, \mathrm{a}+2 \mathrm{~b}\right)$ prototype stretch twin. Acta Cryst. A 2018, 74, 44-53. [CrossRef]

52. Cayron, C.; Logé, R. Evidence of new twinning modes in magnesium questioning the shear paradigm. J. Appl. Cryst. 2018, 51, 809-817.

(C) 2020 by the author. Licensee MDPI, Basel, Switzerland. This article is an open access article distributed under the terms and conditions of the Creative Commons Attribution (CC BY) license (http://creativecommons.org/licenses/by/4.0/). 